MATHEMATICS OF COMPUTATION

Volume 69, Number 230, Pages 691-720

S 0025-5718(99)01144-8

Article electronically published on April 28, 1999

\title{
THE APOLAR BILINEAR FORM IN GEOMETRIC MODELING
}

\author{
GERT VEGTER
}

\begin{abstract}
Some recent methods of Computer Aided Geometric Design are related to the apolar bilinear form, an inner product on the space of homogeneous multivariate polynomials of a fixed degree, already known in 19th century invariant theory. Using a generalized version of this inner product, we derive in a straightforward way some of the recent results in CAGD, like Marsden's identity, the expression for the de Boor-Fix functionals, and recursion schemes for the computation of B-patches and their derivatives.
\end{abstract}

\section{INTRODUCTION}

A common problem in Computer Aided Geometric Design (CAGD) and approximation theory is the construction of suitable bases for the space of piecewise polynomials, defined over simplices in some higher dimensional euclidean space. Several methods have been designed to obtain algorithmically convenient bases. The constraint to work with basis polynomials having local support leads to the construction of the well-known B-splines in the univariate case. Traditionally, these are defined in terms of a recursion scheme, see e.g. de Boor 7] and Schumaker [25]. (There are other, equivalent constructions, like the approach based on finite differences [8], and Schoenberg's geometric construction; see [5] and [17].)

More recent methods employ polarization (also called blossoming), a classical mathematical tool, first introduced into the realm of CAGD by de Casteljau in his seminal work [2], and also [3], and by Ramshaw 21]. These polarization techniques greatly simplified the derivation of many results in the theory of Bézier and B-spline curves. Cavaretta, Dahmen, Micchelli and Seidel [4, 6, 17] succesfully applied the blossoming technique to construct B-patches, which can be considered as local multivariate generalizations of B-splines. Experience with the implementation of this scheme in two dimensions is described in [11. A multivariate generalization of Marsden's equality (see [16] for the original univariate version) plays an important role in the development of a recursion scheme for B-patches. Multivariate versions of the de Boor-Fix functionals have been considered by Lodha and Goldman [14] and [15] to derive some of the important properties of B-patches.

In this paper we relate some of these recent results of computer aided geometric design and constructive approximation to techniques from mathematical disciplines like 19th century invariant theory and differential algebra. The key tool is a generalization of the apolar bilinear form defined on the space of homogeneous polynomials

Received by the editor April 30, 1998.

1991 Mathematics Subject Classification. Primary 41A15, 65D17; Secondary 65D07, 41A63.

Key words and phrases. Apolar bilinear form, polarization, homogeneous polynomials, lineal polynomials, dual basis, Euler's identity, Marsden's identity, Bernstein-Bézier patches, B-patches, de Casteljau, de Boor, recurrence relations, algorithm, basis conversion. 
(of a certain degree, and with a fixed number of variables). This bilinear form, used extensively in the symbolic method of the classical theory of invariants, has been revitalized by Rota and his co-workers, cf. [9] and [13]. A similar binary form on the space of univariate polynomials of a fixed degree has been studied by Goldman [12].

In this paper we introduce a bilinear pairing between the spaces of homogeneous polynomials of degrees $n$ and $m$, where $n \geq m$, with values in the space of polynomials of degree $n-m$. This pairing, introduced in Section 2, coincides with the apolar bilinear form mentioned above in case $n=m$. In Section 3 some simple properties of this pairing are used to derive in a straightforward way some of the recent results in multivariate approximation theory mentioned above. Among these are straightforward proofs of the equivalence of Marsden's equality and duality for any pair of bases of the space of homogeneous polynomials, and the relation of this apolar inner product to the de Boor-Fix functionals. These results are applied to derive well-known results of multivariate Bernstein-Bézier theory, like degree elevation, expressions for derivatives, and recurrence relations for Bernstein polynomials.

In Section 4 we introduce a general criterion for deciding whether a collection of polynomials forms a basis for the space of multivariate polynomials of a fixed degree. These results are applied to study so-called lineal polynomial bases and their dual bases. The B-patch basis is introduced in [6] as the dual of a special lineal basis. Starting from this definition, we almost mechanically obtain the recursion schemes for B-patches and the generalized de Casteljau/de Boor algorithms for evaluation of a polynomial in B-patch form.

In Section 5 we announce future research concerned with applications of the methods of this paper to problems like solving constant coefficient polynomial PDE's, and degree reduction of Bézier patches.

\section{VECTOR SPACES OF FORMS}

2.1. Introduction and terminology. Let $e_{1}, \ldots, e_{s}$ be the standard basis vectors on $\mathbb{R}^{s}$, and let $x=\left(x_{1}, \ldots, x_{s}\right)$ be the standard coordinates on $\mathbb{R}^{s}$. The standard inner product on $\mathbb{R}^{s}$ is denoted by $(\cdot, \cdot)$, i.e., $(u, v)=u_{1} v_{1}+\cdots+u_{s} v_{s}$, for $u, v \in \mathbb{R}^{s}$.

A central object in this paper is the space of real homogeneous polynomials of degree $n$ on $\mathbb{R}^{s}$, denoted by $\mathcal{H}_{n}\left(\mathbb{R}^{s}\right)$. A polynomial in $\mathcal{H}_{n}\left(\mathbb{R}^{s}\right)$ is the sum of monomials of the form $c_{\alpha} x_{1}^{\alpha_{1}} \cdots x_{s}^{\alpha_{s}}$, where $c_{\alpha} \in \mathbb{R}$ and $\alpha=\left(\alpha_{1}, \ldots, \alpha_{s}\right) \in \mathbb{Z}_{\geq 0}^{s}$ is a multi-index of weight $|\alpha|=\alpha_{1}+\cdots+\alpha_{s}$. For convenience the monomial $x_{1}^{\alpha_{1}} \cdots x_{s}^{\alpha_{s}}$ is denoted by $x^{\alpha}$. Linear homogeneous polynomials on $\mathbb{R}^{s}$ are of the form $f(x)=$ $(u, x)$, for some $u \in \mathbb{R}^{s}$. We denote $f$ by $(u, \cdot)$. A lineal polynomial of degree $m$ is the product of $m$ linear polynomials, and hence of the form $\left(u^{1}, \cdot\right) \cdots\left(u^{m}, \cdot\right) \in \mathcal{H}_{m}\left(\mathbb{R}^{s}\right)$, for $u^{1}, \ldots, u^{m} \in \mathbb{R}^{s}$.

For multi-indices $\alpha=\left(\alpha_{1}, \ldots, \alpha_{s}\right)$ and $\beta=\left(\beta_{1}, \ldots, \beta_{s}\right)$ in $\mathbb{Z}_{\geq 0}^{s}$ we define $\alpha \preceq \beta$ iff $\alpha_{i} \leq \beta_{i}$ for $i=1, \cdots, s$. The relation $\preceq$ is a partial order on $\mathbb{Z}_{>0}^{s}$. The set of multi-indices in $\mathbb{Z}_{\geq 0}^{s}$ of weight $n$, denoted by $\Gamma_{s, n}$, is a finite set with

$$
\# \Gamma_{s, n}=\left(\begin{array}{c}
n+s-1 \\
n
\end{array}\right)
$$


elements. For $\alpha \in \Gamma_{s, n}$ the factorial function is defined by $\alpha !=\alpha_{1} ! \cdots \alpha_{s} !$, and the multinomial coefficient $\left(\begin{array}{l}n \\ \alpha\end{array}\right)$ is defined by

$$
\left(\begin{array}{l}
n \\
\alpha
\end{array}\right)=\frac{n !}{\alpha_{1} ! \cdots \alpha_{s} !}
$$

Let $\partial=\left(\partial_{1}, \ldots, \partial_{s}\right)$, with $\partial_{i}=\partial / \partial x_{i}$. With a polynomial $f(x)=\sum_{\alpha \in \Gamma_{s, m}} c_{\alpha} x^{\alpha}$ we associate the homogeneous differential operator $f(\partial)=\sum_{\alpha \in \Gamma_{s, m}} c_{\alpha} \partial^{\alpha}$, where $\partial^{\alpha}=\partial_{1}^{\alpha_{1}} \cdots \partial_{s}^{\alpha_{s}}$. The directional derivative $D_{u}: \mathcal{H}_{n}\left(\mathbb{R}^{s}\right) \rightarrow \mathcal{H}_{n-1}\left(\mathbb{R}^{s}\right)$ with respect to $u \in \mathbb{R}^{s}$ is the differential operator $(u, \partial)$, i.e., $D_{u}=u_{1} \partial_{1}+\cdots+u_{s} \partial_{s}$. Note that $\partial_{i}=\left(e_{i}, \partial\right)=D_{e_{i}}$. Considering $e_{i}$ as a multi-index of weight one, we also have $\partial_{i}=\partial^{e_{i}}$.

2.2. Apolar pairing. This subsection is concerned with a straightforward generalization of the rather well-known apolar inner product $[f, g]=f(\partial) g$, defined on the space of homogeneous polynomials $\mathcal{H}_{n}\left(\mathbb{R}^{s}\right)$. The main result concerns a characterization of this inner product in terms of three simple properties, that will be the basis for the construction of special bases of $\mathcal{H}_{n}\left(\mathbb{R}^{s}\right)$ in later sections.

Definition 2.1. For fixed integers $m$ and $n$, with $0 \leq m \leq n$, the apolar pairing is the map

$$
[\cdot, \cdot]_{m, n}: \mathcal{H}_{m}\left(\mathbb{R}^{s}\right) \times \mathcal{H}_{n}\left(\mathbb{R}^{s}\right) \rightarrow \mathcal{H}_{n-m}\left(\mathbb{R}^{s}\right),
$$

associating to the homogeneous polynomials $f \in \mathcal{H}_{m}\left(\mathbb{R}^{s}\right)$ and $g \in \mathcal{H}_{n}\left(\mathbb{R}^{s}\right)$ the homogeneous polynomial $[f, g]_{m, n}$ of degree $n-m$, defined by

$$
[f, g]_{m, n}=\frac{(n-m) !}{n !} f(\partial) g .
$$

The constant $(n-m) ! / n$ ! in the right hand side of the latter identity is chosen in such a way that apolar pairing is a reproducing kernel, cf. Corollary 2.3.

Note that we have in fact a family of pairings, one for each pair of integers $m$ and $n$ with $0 \leq m \leq n$. In this paper, the term pairing refers to the whole family of bilinear maps. From now on we shall drop the subscripts $m$ and $n$, since they are implicitly known as the degree of the first and second argument of the pairing operator.

Theorem 2.2. The apolar pairing is the unique bilinear pairing with the following properties.

1. Apolar pairing with constants. For $f \in \mathcal{H}_{n}\left(\mathbb{R}^{s}\right)$ :

$$
[1, f]=f,
$$

where $1 \in \mathcal{H}_{0}\left(\mathbb{R}^{s}\right)$ is the constant homogeneous polynomial of degree 0 .

2. Apolar pairing with linear forms. For $f \in \mathcal{H}_{n}\left(\mathbb{R}^{s}\right)$ and $u \in \mathbb{R}^{s}$ :

$$
[(u, \cdot), f]=\frac{1}{n} D_{u} f .
$$

3. Transposition of a homogeneous factor. For $f_{1} \in \mathcal{H}_{m_{1}}\left(\mathbb{R}^{s}\right), f_{2} \in$ $\mathcal{H}_{m_{2}}\left(\mathbb{R}^{s}\right)$, and $g \in \mathcal{H}_{n}\left(\mathbb{R}^{s}\right)$, with $m_{1}+m_{2} \leq n$ :

$$
\left[f_{1} f_{2}, g\right]=\left[f_{1},\left[f_{2}, g\right]\right] \text {. }
$$


Proof. It is obvious that apolar pairing is a bilinear operator, satisfying properties 1,2 and 3 . We prove uniqueness using mathematical induction. So assume that $[\cdot, \cdot]$ is a bilinear pairing satisfying properties 1,2 and 3 ; then we need to prove that $[f, g]=\frac{(n-m) !}{n !} f(\partial) g$. To this end, let $P(m)$ be the predicate:

For all integers $n$, with $m \leq n$ : if $f \in \mathcal{H}_{m}\left(\mathbb{R}^{s}\right)$ and $g \in \mathcal{H}_{n}\left(\mathbb{R}^{s}\right)$,

$$
\text { then }[f, g]=\frac{(n-m) !}{n !} f(\partial) g \text {. }
$$

Obviously, property 1 implies that $P(0)$ holds. So consider $m>0$, and assume that $P(k)$ holds for $0 \leq k<m$. To express the pairing $[f, g]$ in terms of pairings of homogeneous polynomials of lower degree, we use Euler's identity for homogeneous polynomials to rewrite $f \in \mathcal{H}_{m}\left(\mathbb{R}^{s}\right)$ as

$$
f=\frac{1}{m} \sum_{i=1}^{s}\left(e_{i}, \cdot\right) \partial_{i} f
$$

Using the fact that $\left(e_{i}, \partial\right)=\partial_{i}$ we get

$$
f(\partial) g=\left(\frac{1}{m} \sum_{i=1}^{s} \partial_{i} f(\partial)\right)\left(\partial_{i} g\right) .
$$

On the other hand, using (2.1) and properties 2 and 3 of the pairing operator, we see that

$$
[f, g]=\frac{1}{m} \sum_{i=1}^{s}\left[\partial_{i} f,\left[\left(e_{i}, \cdot\right), g\right]\right]=\frac{1}{m} \sum_{i=1}^{s} \frac{1}{n}\left[\partial_{i} f, \partial_{i} g\right] .
$$

Since $\partial_{i} f \in \mathcal{H}_{m-1}\left(\mathbb{R}^{s}\right)$, we may apply the induction hypothesis $P(m-1)$ to the bracket in the latter expression, yielding

$$
\left[\partial_{i} f, \partial_{i} g\right]=\frac{(n-m) !}{(n-1) !} \partial_{i} f(\partial)\left(\partial_{i} g\right) .
$$

Hence $P(m)$ follows from (2.2), (2.3) and (2.4). This completes the uniqueness part of the proof.

Using the defining properties 1, 2 and 3 of the main theorem we obtain the following simple result concerning the pairing with polynomials of a special form:

Corollary 2.3. 1. Apolar pairing with a lineal polynomial. Let $u^{1}, \ldots, u^{m} \in$ $\mathbb{R}^{s}$, and $f \in \mathcal{H}_{n}\left(\mathbb{R}^{s}\right)$, with $0 \leq m \leq n$. Then

$$
\left[\left(u^{1}, \cdot\right) \cdots\left(u^{m}, \cdot\right), f\right]=\frac{(n-m) !}{n !} D_{u^{1}} \cdots D_{u^{m}} f .
$$

2. Apolar pairing is a reproducing kernel. For $f \in \mathcal{H}_{n}\left(\mathbb{R}^{s}\right)$ and $y \in \mathbb{R}^{s}$,

$$
\left[f,(y, \cdot)^{n}\right]=f(y) \text {. }
$$

3. Apolar pairing with the power of a linear form. For $f \in \mathcal{H}_{m}\left(\mathbb{R}^{s}\right)$, $y \in \mathbb{R}^{s}$, and $0 \leq m \leq n$,

$$
\left[f,(y, \cdot)^{n}\right]=f(y)(y, \cdot)^{n-m} .
$$


Proof. 1. Since $\left(u^{1}, \partial\right) \cdots\left(u^{m}, \partial\right)=D_{u^{1}} \cdots D_{u^{m}}$, the first property follows from the definition of apolar pairing.

2. Identity (2.6) obviously holds for $n=0$. So assume, inductively, it holds for $n-1$, where $n>0$. Using properties 2 and 3 from Theorem 2.2 we see that $\left[f,(y, \cdot)^{n}\right]=\frac{1}{n}\left[(y, .)^{n-1}, D_{y} f\right]$. Using the induction hypothesis yields

$$
\frac{1}{n}\left[(y, .)^{n-1}, D_{y} f\right]=\frac{1}{n} D_{y} f(y)=\frac{1}{n} \sum_{i=1}^{s} y_{i} \partial_{i} f(y)=f(y),
$$

by Euler's identity for homogeneous polynomials of degree $n$.

3. In the special case $f=(z, \cdot)^{m}$, for $z \in \mathbb{R}^{s}$, the identity follows by induction, where we repeatedly use properties 2 and 3 from Theorem 2.2 together with $D_{z}(y, \cdot)^{k}=k(y, z)(y, \cdot)^{k-1}$. To prove the identity for general $f \in \mathcal{H}_{m}\left(\mathbb{R}^{s}\right)$, consider the polynomial $g=\left[f,(y, \cdot)^{n}\right] \in \mathcal{H}_{n-m}\left(\mathbb{R}^{s}\right)$. Since we have already proven that apolar pairing is a reproducing kernel, we see that, for $z \in \mathbb{R}^{s}$ :

$$
\begin{aligned}
g(z) & =\left[(z, \cdot)^{n-m}, g\right]=\left[f,\left[(z, \cdot)^{n-m},(y, \cdot)^{n}\right]\right] \\
& =(y, z)^{n-m}\left[f,(y, \cdot)^{m}\right]=(y, z)^{n-m} f(y) .
\end{aligned}
$$

Identifying the space of zero degree polynomials with $\mathbb{R}$, we see that, for $n=m$, apolar pairing corresponds to a real bilinear form on the space of homogeneous polynomials of degree $m$. The next result states that this bilinear form is even an inner product.

Proposition 2.4. The apolar bilinear form $[\cdot, \cdot]: \mathcal{H}_{m}\left(\mathbb{R}^{s}\right) \times \mathcal{H}_{m}\left(\mathbb{R}^{s}\right) \rightarrow \mathbb{R}$ is an inner product on the space of homogeneous polynomials of degree $m$.

Proof. Bilinearity and symmetry of the pairing are obvious from the definition. Since $f(\partial) f=\sum_{\alpha \in \Gamma_{s, m}} \frac{1}{\alpha !}\left(\partial^{\alpha} f\right)^{2}$, we see that $[f, f] \geq 0$ for all $f \in \mathcal{H}_{m}\left(\mathbb{R}^{s}\right)$. Furthermore $[f, f]=0$ implies $\partial^{\alpha} f=0$, for all $\alpha \in \Gamma_{s, m}$, so $f=0$. Therefore the bilinear form is positive definite.

To prove that a polynomial $f \in \mathcal{H}_{m}\left(\mathbb{R}^{s}\right)$ is the zero-polynomial we just have to show that $[f, g]=0$ for all $g \in \mathcal{H}_{m}\left(\mathbb{R}^{s}\right)$. This follows directly from Proposition 2.4 The next result is an extension of this observation to the case in which the degrees of $f$ and $g$ are not necessarily equal.

Lemma 2.5. Let $0 \leq m \leq n$.

1. Let $f \in \mathcal{H}_{m}\left(\mathbb{R}^{s}\right)$. If $[f, g]=0$, for all $g \in \mathcal{H}_{n}\left(\mathbb{R}^{s}\right)$, then $f=0$.

2. Let $g \in \mathcal{H}_{n}\left(\mathbb{R}^{s}\right)$. If $[f, g]=0$, for all $f \in \mathcal{H}_{m}\left(\mathbb{R}^{s}\right)$, then $g=0$.

Proof. 1. For $y \in \mathbb{R}^{s}$ we have $\left[f,(y, \cdot)^{n}\right]=0 \in \mathcal{H}_{n-m}\left(\mathbb{R}^{s}\right)$. By Corollary 2.3 $\left[f,(y, \cdot)^{n}\right]=f(y)(y, \cdot)^{n-m}$, so $f(y)=0$ for all $y \in \mathbb{R}^{s} \backslash\{0\}$. Therefore $f=0$.

2. For all $y \in \mathbb{R}^{s}$ we have $g(y)=\left[(y, \cdot)^{n}, g\right]=\left[(y, \cdot)^{n-m},\left[(y, \cdot)^{m}, g\right]\right]=0$, so $g=0$. 
As an immediate consequence we have the important spanning property; See also Reznick [23]. Rephrased loosely, it allows us to conclude that all polynomials in $\mathcal{H}_{m}\left(\mathbb{R}^{s}\right)$ have a certain property if the property holds for all $m$-th powers of linear forms. More precisely:

Lemma 2.6 (Spanning property of powers of linear forms). Let $O \subset \mathbb{R}^{s}$ be an open subset of $\mathbb{R}^{s}$. Then the set $\left\{(y, \cdot)^{m} \mid y \in O\right\}$ spans the space of homogeneous polynomials of degree $m$.

Proof. We first prove the result in case $O=\mathbb{R}^{s}$. Let $U$ be the linear subspace of $\mathcal{H}_{m}\left(\mathbb{R}^{s}\right)$ spanned by the $m$-th powers $\left\{(y, \cdot)^{m} \mid y \in \mathbb{R}^{s}\right\}$. Then $U^{\perp}=\{f \in$ $\mathcal{H}_{m}\left(\mathbb{R}^{s}\right) \mid\left[(y, \cdot)^{m}, f\right]=0$ for all $\left.y \in \mathbb{R}^{s}\right\}$, so $f \in U^{\perp}$ iff $f(y)=0$ for all $y \in$ $\mathbb{R}^{s}$ according to Corollary 2.3 2. This implies $U^{\perp}=\{0\}$. As we have seen in Proposition 2.4 apolar pairing is an inner product in case $n=m$, so $U=\mathcal{H}_{m}\left(\mathbb{R}^{s}\right)$.

To prove the general case, let $Y_{M}$ be a finite subset of $\mathbb{R}^{s}$ such that $\left\{(y, \cdot)^{m} \mid\right.$ $\left.y \in Y_{M}\right\}$ is a basis for $\mathcal{H}_{m}\left(\mathbb{R}^{s}\right)$. Such a set exists in view of the first part of the proof. Furthermore, $\# Y_{M}=\# \Gamma_{s, m}$. Let $A$ be a non-singular $s \times s$ matrix such that $A y \in O$, for all $y \in Y_{M}$. For $f \in \mathcal{H}_{m}\left(\mathbb{R}^{s}\right)$ consider the homogenous $m$-th degree polynomial $f\left(\left(A^{T}\right)^{-1} x\right)$. There are real constants $c_{y}, y \in Y_{M}$, such that $f\left(\left(A^{T}\right)^{-1} x\right)=\sum_{y \in Y_{M}}(y, x)^{m}$. The polynomial $f$ can now be expressed as a linear combination of powers of linear forms as follows:

$$
f(x)=\sum_{y \in Y_{M}} c_{y}\left(y, A^{T} x\right)^{m}=\sum_{y \in Y_{M}} c_{y}(A y, x)^{m}=\sum_{y \in \bar{Y}_{M}} \bar{c}_{y}(y, x)^{m},
$$

where $\bar{Y}_{M}=\left\{A y \mid y \in Y_{M}\right\} \subset O$, and $\bar{c}_{y}=c_{A^{-1} y} \in \mathbb{R}$. This completes the proof of the general case.

One can in fact prove that the set $\left\{(\alpha, \cdot)^{n} \mid \alpha \in \Gamma_{s, n}\right\}$ is a basis for $\mathcal{H}_{n}\left(\mathbb{R}^{s}\right)$. This is a generalization of a theorem of Biermann, who proved it for the case $s=3$; see Reznick [23 Proposition 2.11].

2.3. Apolar pairing and polar forms. Ramshaw [21] uses the technique of polarization (also called blossoming) to obtain a very elegant definition of univariate B-splines. This technique is based on the identification of the space of homogeneous polynomials with the space $\mathcal{S}^{n}\left(\mathbb{R}^{s}\right)$ of symmetric $n$-linear real forms on $\mathbb{R}^{s}$. More precisely, consider the diagonal map diag : $\mathcal{S}^{n}\left(\mathbb{R}^{s}\right) \rightarrow \mathcal{H}_{n}\left(\mathbb{R}^{s}\right)$, defined for the symmetric $n$-linear form $F: \mathbb{R}^{s} \times \cdots \times \mathbb{R}^{s} \rightarrow \mathbb{R}$ by

$$
(\operatorname{diag} F)(x)=F(\underbrace{x, \ldots, x}_{n}) .
$$

Obviously $\operatorname{diag} F \in \mathcal{H}_{n}\left(\mathbb{R}^{s}\right)$. We shall refer to it as the diagonal of $F$. In fact, the diagonal map is an isomorphism of vector spaces, as we shall prove presently. Its inverse is the well-known polarization operator, defined as follows.

Definition 2.7. The polarization operator $\mathcal{P}^{n}$ maps the homogeneous polynomial $f \in \mathcal{H}_{n}\left(\mathbb{R}^{s}\right)$ to the $n$-linear form $\mathcal{P}^{n} f$ defined by

$$
\mathcal{P}^{n} f\left(\xi^{(1)}, \ldots, \xi^{(n)}\right)=\frac{1}{n !} D_{\xi^{(1)}} \cdots D_{\xi^{(n)}} f .
$$


Example: Polar form of a bivariate polynomial. From the definition it is obvious that the polarization operator is easily implemented in a computer algebra system like Maple or Mathematica. Doing so for, e.g., the fourth degree homogeneous polynomial $f$ on $\mathbb{R}^{2}$, defined, for $x=\left(x_{1}, x_{2}\right) \in \mathbb{R}^{2}$, by $f(x)=x_{1}^{2} x_{2}^{2}$, we obtain

$$
\begin{aligned}
\mathcal{P}^{4} f\left(\xi^{(1)}, \xi^{(2)}, \xi^{(3)}, \xi^{(4)}\right)= & \frac{1}{6}\left(\xi_{2}^{(1)} \xi_{2}^{(2)} \xi_{1}^{(3)} \xi_{1}^{(4)}+\xi_{2}^{(1)} \xi_{1}^{(2)} \xi_{2}^{(3)} \xi_{1}^{(4)}+\xi_{1}^{(1)} \xi_{2}^{(2)} \xi_{2}^{(3)} \xi_{1}^{(4)}\right. \\
& \left.+\xi_{2}^{(1)} \xi_{1}^{(2)} \xi_{1}^{(3)} \xi_{2}^{(4)}+\xi_{1}^{(1)} \xi_{2}^{(2)} \xi_{1}^{(3)} \xi_{2}^{(4)}+\xi_{1}^{(1)} \xi_{1}^{(2)} \xi_{2}^{(3)} \xi_{2}^{(4)}\right)
\end{aligned}
$$

Taking $\xi^{(i)}=x$, for $i=1, \ldots, 4$, it is easy to check that we recover the polynomial $f$, i.e., $\mathcal{P}^{4} f(x, x, x, x)=x_{1}^{2} x_{2}^{2}=f(x)$.

A straightforward application of Corollary 2.3 shows that the polar form of a polynomial is just the apolar pairing of the polynomial and a lineal polynomial:

Proposition 2.8. For $f \in \mathcal{H}_{n}\left(\mathbb{R}^{s}\right)$ and $\xi^{1}, \ldots, \xi^{n} \in \mathbb{R}^{s}$ :

$$
\mathcal{P}^{n} f\left(\xi^{1}, \ldots, \xi^{n}\right)=\left[\left(\xi^{1}, \cdot\right) \cdots\left(\xi^{n}, \cdot\right), f\right] .
$$

In particular, $\mathcal{P}^{n} f(\underbrace{y, \ldots, y}_{n})=f(y)$, for $y \in \mathbb{R}^{s}$.

As announced, diagonalization and polarization are inverse to one another. More precisely:

Proposition 2.9. The polarization operator $\mathcal{P}^{n}$ is an isomorphism between the linear spaces $\mathcal{H}_{n}\left(\mathbb{R}^{s}\right)$ and $\mathcal{S}^{n}\left(\mathbb{R}^{s}\right)$. Its inverse is the diagonal map.

Proof. In view of Proposition 2.8 the polarization operator $\mathcal{P}^{n}$ maps a homogeneous polynomial to a symmetric $n$-linear form, so its image is indeed contained in the space $\mathcal{S}^{n}\left(\mathbb{R}^{s}\right)$. The second part of Proposition 2.8 can be rephrased by saying that $\mathcal{P}^{n}$ is a right inverse of $\operatorname{diag}: \mathcal{S}^{n}\left(\mathbb{R}^{s}\right) \rightarrow \mathcal{H}_{n}\left(\mathbb{R}^{s}\right)$. From this we conclude that the diagonal map is injective. Therefore, to show that it is an isomorphism, it is sufficient to prove that

$$
\operatorname{dim} \mathcal{S}^{n}\left(\mathbb{R}^{s}\right) \leq \operatorname{dim} \mathcal{H}_{n}\left(\mathbb{R}^{s}\right) .
$$

This inequality follows from the observation that a symmetric $n$-linear map $F$ is uniquely determined by the values

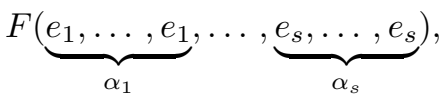

where $\alpha=\left(\alpha_{1}, \ldots, \alpha_{s}\right)$ ranges over $\Gamma_{s, n}$. Therefore $\operatorname{dim} \mathcal{S}^{n}\left(\mathbb{R}^{s}\right) \leq \# \Gamma_{s, n}=$ $\operatorname{dim} \mathcal{H}_{n}\left(\mathbb{R}^{s}\right)$.

Finally, the apolar pairing of two polynomials can be defined in terms of their polar forms. In case the paired polynomials have the same degree, this definition coincides with the definition of Beauzamy et al. [1].

Proposition 2.10. Let $f \in \mathcal{H}_{m}\left(\mathbb{R}^{s}\right)$ and $g \in \mathcal{H}_{n}\left(\mathbb{R}^{s}\right)$, where $0 \leq m \leq n$. Then

$$
[f, g](x)=\sum_{i_{1}, \ldots, i_{m}=1}^{s} \mathcal{P}^{m} f\left(e_{i_{1}}, \ldots, e_{i_{m}}\right) \mathcal{P}^{n} g(e_{i_{1}}, \ldots, e_{i_{m}}, \underbrace{x, \ldots, x}_{n-m}) .
$$


Proof. Denote the right hand side of this identity by $\sigma(f, g)$. Obviously, $\sigma$ is a bilinear map $\mathcal{H}_{m}\left(\mathbb{R}^{s}\right) \times \mathcal{H}_{n}\left(\mathbb{R}^{s}\right) \rightarrow \mathcal{H}_{n-m}\left(\mathbb{R}^{s}\right)$. In view of the spanning property, viz Lemma [2.6] it is sufficient to prove that $\sigma\left(f,(y, \cdot)^{n}\right)=\left[f,(y, \cdot)^{n}\right]$, for all $y \in \mathbb{R}^{s}$. So let $g=(y, \cdot)^{n}$; then $\mathcal{P}^{n} g\left(\xi^{1}, \ldots, \xi^{n}\right)=\left(y, \xi^{1}\right) \cdots\left(y, \xi^{n}\right)$. This follows readily from Proposition 2.9. in view of the observation that both sides of the latter identity are symmetric $n$-linear forms on $\mathbb{R}^{s}$, that coincide upon diagonalization. In particular

$$
\mathcal{P}^{n} g(e_{i_{1}}, \ldots, e_{i_{m}}, \underbrace{x, \ldots, x}_{n-m})=y_{i_{1}} \cdots y_{i_{m}}(y, x)^{n-m}
$$

and hence the result follows from the following derivation:

$$
\begin{aligned}
\sigma(f, g)(x) & =\sum_{i_{1}, \ldots, i_{m}=1}^{s} \mathcal{P}^{m} f\left(e_{i_{1}}, \ldots, e_{i_{m}}\right) y_{i_{1}} \cdots y_{i_{m}}(y, x)^{n-m} \\
& =\sum_{i_{1}, \ldots, i_{m}=1}^{s} \mathcal{P}^{m} f\left(y_{i_{1}} e_{i_{1}}, \ldots, y_{i_{m}} e_{i_{m}}\right)(y, x)^{n-m} \\
& =\mathcal{P}^{m} f(\underbrace{y, \ldots, y}_{m})(y, x)^{n-m} \\
& =f(y)(y, x)^{n-m} \\
& =[f, g](x) .
\end{aligned}
$$

\section{DuAl BASES}

Dual bases, the topic of this section, are the second key tool for the construction of computationally convenient polynomial bases. In CAGD dual bases have been used successfully in the construction of special bases, like the B-spline basis for the space of piecewise polynomials. Recursive expressions (with respect to the degree of the basis functions) are derived from Marsden's identity; see, e.g., 4] and [17].

First it is shown, in Section 3.1 that two bases form a dual pair iff they satisfy Marsden's identity. Section [3.3] starts with Euler's identity for the decomposition of a polynomial of degree $n$ with respect to a basis of degree $m$ in case the dual of the latter basis is given. This identity is the basis for the development of recursive algorithms for dual bases in later sections.

3.1. Dual bases and Marsden's identity. In CAGD, the notion of dual bases is slightly different from the usual notion in linear algebra. Therefore we recall the definition of a dual basis pair with respect to the apolar inner product $[\cdot, \cdot]$ on $\mathcal{H}_{m}\left(\mathbb{R}^{s}\right)$.

Definition 3.1. The dual basis of a basis $\left\{f_{\alpha} \mid \alpha \in \Gamma_{s, m}\right\}$ of $\mathcal{H}_{m}\left(\mathbb{R}^{s}\right)$ is a collection $\left\{g_{\alpha} \mid \alpha \in \Gamma_{s, m}\right\}$ of polynomials in $\mathcal{H}_{m}\left(\mathbb{R}^{s}\right)$ such that, for $\alpha, \beta \in \Gamma_{s, m}$,

$$
\left[f_{\alpha}, g_{\beta}\right]=\delta_{\alpha \beta}
$$

It is an easy to prove standard fact from linear algebra that a dual basis is indeed a basis. Given a dual basis pair $(\mathcal{F}, \mathcal{G})$, a polynomial $f \in \mathcal{H}_{m}\left(\mathbb{R}^{s}\right)$ can be expressed 
with respect to either basis in terms of coefficients depending on the other one:

$$
f=\sum_{\alpha \in \Gamma_{s, m}}\left[g_{\alpha}, f\right] f_{\alpha}=\sum_{\alpha \in \Gamma_{s, m}}\left[f_{\alpha}, f\right] g_{\alpha} .
$$

It should be noted that traditionally the dual basis of $\mathcal{F}$ is a basis $\left\{\lambda_{\alpha} \mid \alpha \in \Gamma_{s, m}\right\}$ for the space of linear functionals on $\mathcal{H}_{m}\left(\mathbb{R}^{s}\right)$, such that $\lambda_{\alpha}\left(f_{\beta}\right)=\delta_{\alpha \beta}$. Obviously, in the context of Definition 3.1 these linear functionals are defined by $\lambda_{\alpha}(f)=\left[g_{\alpha}, f\right]$. With the help of this collection of linear functionals we can express any $f \in \mathcal{H}_{n}\left(\mathbb{R}^{s}\right)$ with respect to the basis $\mathcal{F}$ as $f=\sum_{\alpha \in \Gamma_{s, n}} \lambda_{\alpha}(f) f_{\alpha}$. In Section 4.1 we introduce the multivariate B-patch basis and its dual. There it will become clear that the functionals $\lambda_{\alpha}$, defined with respect to this B-patch basis and its dual, are equalup to a constant - to the de Boor-Fix functionals. See also [14].

Proposition 3.2. Let $\mathcal{F}=\left\{f_{\alpha} \mid \alpha \in \Gamma_{s, m}\right\}$ and $\mathcal{G}=\left\{g_{\alpha} \mid \alpha \in \Gamma_{s, m}\right\}$ be two collections of polynomials in $\mathcal{H}_{m}\left(\mathbb{R}^{s}\right)$. Then the following statements are equivalent:

1. $\mathcal{F}$ and $\mathcal{G}$ are dual bases with respect to the apolar pairing on $\mathcal{H}_{m}\left(\mathbb{R}^{s}\right)$.

2. Marsden's identity. For $x, y \in \mathbb{R}^{s}$ :

$$
(x, y)^{m}=\sum_{\alpha \in \Gamma_{s, m}} f_{\alpha}(x) g_{\alpha}(y) .
$$

Proof. First assume that $\mathcal{F}$ and $\mathcal{G}$ are dual bases. Since apolar pairing is a reproducing kernel, we have $\left[f_{\alpha},(x, \cdot)^{m}\right]=f_{\alpha}(x)$. Therefore $\left(\underline{3.1}\right.$, with $f=(x, \cdot)^{m}$, yields

$$
(x, \cdot)^{m}=\sum_{\alpha \in \Gamma_{s, m}} f_{\alpha}(x) g_{\alpha},
$$

which is equivalent to Marsden's identity.

Conversely, assume that Marsden's identity, or, equivalently, identity (3.2) holds. In view of the spanning property, Lemma 2.6 this implies that $\mathcal{G}$ spans $\mathcal{H}_{m}\left(\mathbb{R}^{s}\right)$. Since $\mathcal{G}$ contains $\operatorname{dim} \mathcal{H}_{m}\left(\mathbb{R}^{s}\right)$ elements, $\mathcal{G}$ is even a basis of $\mathcal{H}_{m}\left(\mathbb{R}^{s}\right)$. By symmetry, the same holds for $\mathcal{F}$. Apolar pairing of both sides of 3.2 with $f_{\beta}$ yields

$$
f_{\beta}(x)=\left[f_{\beta},(x, \cdot)^{m}\right]=\sum_{\alpha \in \Gamma_{s, m}} f_{\alpha}(x)\left[f_{\beta}, g_{\alpha}\right] .
$$

Since $\mathcal{F}$ is a basis, it follows that $\left[f_{\beta}, g_{\alpha}\right]=\delta_{\beta \alpha}$. This completes the proof.

\subsection{The Bernstein-Bézier basis.}

The homogeneous Bernstein-Bézier basis. As an instructive application we use Proposition 3.2 to determine the dual of the homogeneous Bernstein-Bézier basis of $\mathcal{H}_{n}\left(\mathbb{R}^{s}\right)$. This example will serve to illustrate that many familiar results from the theory of multivariate Bernstein-Bézier patches are straightforward consequences of the rather general theory developed in later sections. Furthermore, it is also our starting point for the generalization of the Bernstein-Bézier basis, thereby constructing the B-patch basis. Here we follow the approach of [6] and [17].

Let $\left\{x^{1}, \ldots, x^{s}\right\}$ be a basis of $\mathbb{R}^{s}$, and let the set of linear forms $\left\{u_{1}, \ldots, u_{s}\right\}$ on $\mathbb{R}^{s}$ be its dual basis in the usual sense, i.e., $u_{i}\left(x_{j}\right)=\delta_{i j}$. In view of the reproducing kernel property, the basis of linear forms is dual, in the sense of Definition [3.1, to 
the basis $\left\{\left(x^{1}, \cdot\right), \ldots,\left(x^{s}, \cdot\right)\right\}$ of $\mathcal{H}_{1}\left(\mathbb{R}^{s}\right)$. Since every $x \in \mathbb{R}^{s}$ can be written as

$$
x=\sum_{i=1}^{s} u_{i}(x) x^{i}
$$

we see that, for $x, y \in \mathbb{R}^{s}$,

$$
(x, y)=\sum_{i=1}^{s} u_{i}(x)\left(x^{i}, y\right) .
$$

(This is, in fact, Marsden's identity on $\mathcal{H}_{1}\left(\mathbb{R}^{s}\right)$.) Taking $n$-th powers of both sides of the last equality, and using the multinomial theorem, one readily checks that

$$
(x, y)^{n}=\sum_{\alpha \in \Gamma_{s, n}} B_{\alpha}(x) l_{\alpha}(y),
$$

where, for $\alpha \in \Gamma_{s, n}$, the polynomials

$$
B_{\alpha}(x)=\left(\begin{array}{l}
n \\
\alpha
\end{array}\right) u_{1}(x)^{\alpha_{1}} \cdots u_{s}(x)^{\alpha_{s}}
$$

form the homogeneous Bernstein-Bézier basis of $\mathcal{H}_{n}\left(\mathbb{R}^{s}\right)$ with respect to the basis $\left\{x^{1}, \ldots, x^{s}\right\}$ of $\mathbb{R}^{s}$, whereas

$$
l_{\alpha}(y)=\left(x^{1}, y\right)^{\alpha_{1}} \cdots\left(x^{s}, y\right)^{\alpha_{s}}
$$

are the lineal polynomials with respect to that basis. Now Proposition 3.2 implies that these lineal polynomials form a basis of $\mathcal{H}_{n}\left(\mathbb{R}^{s}\right)$, as $\alpha$ ranges over $\Gamma_{s, n}$, which is the dual basis of the Bernstein-Bézier basis.

The following simple lemma states necessary and sufficient conditions for the orthogonality of the Bernstein-Bézier basis with respect to the apolar inner product.

Lemma 3.3. Let $n \geq 1$, and let the lineal polynomials $l_{\nu}$ and Bernstein-Bézier polynomials $B_{\nu}, \nu \in \Gamma_{s, n}$, be defined with respect to the basis $\left\{x^{1}, \ldots, x^{s}\right\}$ of $\mathbb{R}^{s}$. The following statements are equivalent:

1. $B_{\nu}=\left(\begin{array}{l}n \\ \nu\end{array}\right) l_{\nu}$, for all $\nu \in \Gamma_{s, n}$

2. $\left[l_{\mu}, l_{\nu}\right]=\left(\begin{array}{l}n \\ \mu\end{array}\right)^{-1} \delta_{\mu \nu}$, for all $\mu, \nu \in \Gamma_{s, n}$

3. $\left[B_{\mu}, B_{\nu}\right]=\left(\begin{array}{l}n \\ \mu\end{array}\right) \delta_{\mu \nu}$, for all $\mu, \nu \in \Gamma_{s, n}$

4. $\left\{x^{1}, \ldots, x^{s}\right\}$ is orthonormal with respect to the inner product $(\cdot, \cdot)$.

Proof. The equivalence of the first three claims follows from the definition of duality. To prove that the second claim implies the fourth one, observe that

$$
\left(x^{i}, x^{j}\right)^{n}=\left[\left(x^{i}, \cdot\right)^{n},\left(x^{j}, \cdot\right)^{n}\right]=\left[l_{n e_{i}}, l_{n e_{j}}\right]=\delta_{i j} .
$$

Therefore $\left(x^{i}, x^{j}\right)=\delta_{i j}$, i.e., the basis $\left\{x^{1}, \ldots, x^{s}\right\}$ is orthonormal. Finally, if the fourth claim holds, then $u_{i}(x)=\left(x^{i}, x\right)$, so $B_{\nu}=\left(\begin{array}{l}n \\ \nu\end{array}\right) l_{\nu}$, and hence the first claim holds. This completes the proof of the lemma.

Homogenization and the affine Bernstein-Bézier basis. Let $\mathcal{P}_{n}\left(\mathbb{R}^{s-1}\right)$ denote the space of polynomials over $\mathbb{R}^{s-1}$ of degree at most $n$. Consider the degree- $n$ homogenization operator $H_{n}: \mathcal{P}_{n}\left(\mathbb{R}^{s-1}\right) \rightarrow \mathcal{H}_{n}\left(\mathbb{R}^{s}\right)$, associated with a non-zero linear form $p$ on $\mathbb{R}^{s}$, defined by

$$
H_{n} f(x)=p(x)^{n} f\left(x_{1} / p(x), \ldots, x_{s-1} / p(x)\right),
$$


for $f \in \mathcal{P}_{n}\left(\mathbb{R}^{s-1}\right)$ and $x=\left(x_{1}, \ldots, x_{s}\right) \in \mathbb{R}^{s}$. If $f \in \mathcal{P}_{m}\left(\mathbb{R}^{s-1}\right)$ and $n \geq m$, then

$$
H_{n} f(x)=p(x)^{n-m} H_{m} f(x) .
$$

Suppose $p(x)=1$ has solution $x_{s}=q\left(x_{1}, \ldots, x_{s-1}\right)$. Then the inverse of $H_{n}$ is the operator $K_{n}: \mathcal{H}_{n}\left(\mathbb{R}^{s}\right) \rightarrow \mathcal{P}_{n}\left(\mathbb{R}^{s-1}\right)$, defined by

$$
K_{n} F\left(x_{1}, \ldots, x_{s-1}\right)=F\left(x_{1}, \ldots, x_{s-1}, q\left(x_{1}, \ldots, x_{s-1}\right)\right) .
$$

Below we take $p(x)=x_{1}+\cdots+x_{s}$, so $q\left(x_{1}, \ldots, x_{s-1}\right)=1-x_{1}-\cdots-x_{s-1}$.

The monomial basis of $\mathcal{P}_{n}\left(\mathbb{R}^{s-1}\right)$ is formed by the monomials $x^{\mu}$, with $\mu$ ranging over the union $\Gamma_{s-1, \leq n}:=\Gamma_{s-1,0} \cup \cdots \cup \Gamma_{s-1, n}$. The affine Bernstein-Bézier basis of $\mathcal{P}_{n}\left(\mathbb{R}^{s-1}\right)$ is defined as follows. For $\gamma \in \Gamma_{s-1, m}$, with $0 \leq m \leq n$, let the Bernstein-Bézier polynomial $B_{\gamma}^{n} \in \mathcal{P}_{n}\left(\mathbb{R}^{s-1}\right)$ of degree $n$ be defined by

$$
B_{\gamma}^{n}(x)=K_{n} B_{\gamma+(n-m) e_{s}},
$$

or, equivalently,

$$
B_{\gamma}^{n}(x)=B_{\left(\gamma_{1}, \ldots, \gamma_{s-1}, n-m\right)}\left(x_{1}, \ldots, x_{s-1}, 1-x_{1}-\cdots-x_{s-1}\right) .
$$

Here $B_{\gamma+(n-m) e_{s}} \in \mathcal{H}_{n}\left(\mathbb{R}^{s}\right)$ is the homogeneous Bernstein-polynomial associated with the multi-index $\gamma_{1} e_{1}+\ldots+\gamma_{s-1} e_{s-1}+(n-m) e_{s} \in \Gamma_{s, n}$. For instance, taking $s=2$, we obtain, for $0 \leq m \leq n$, the familiar univariate Bernstein polynomials:

$$
B_{m}^{n}(x)=K_{n} B_{(m, n-m)}(x, 1-x)=\left(\begin{array}{c}
n \\
m
\end{array}\right) x^{m}(1-x)^{n-m} .
$$

Since $K_{n}$ is an isomorphism between $\mathcal{H}_{n}\left(\mathbb{R}^{s}\right)$ and $\mathcal{P}_{n}\left(\mathbb{R}^{s-1}\right)$, the Bernstein-Bézier polynomials $B_{\gamma}^{n}$, where $\gamma$ ranges over $\Gamma_{s-1, \leq n}$, indeed form a basis of $\mathcal{P}_{n}\left(\mathbb{R}^{s-1}\right)$.

Finally, the barycentric Bernstein-Bézier form (of degree $n$ ) of a polynomial $f \in \mathcal{P}_{n}\left(\mathbb{R}^{s-1}\right)$ is the decomposition

$$
f=\sum_{\nu \in \Gamma_{s, n}} c_{\nu} B_{\nu_{1}, \ldots, \nu_{s-1}}^{n}
$$

where the $c_{\nu}$ are the coefficients of $F=H_{n} f \in \mathcal{H}_{n}\left(\mathbb{R}^{s}\right)$ with respect to the homogeneous Bernstein-Bézier basis of $\mathcal{H}_{n}\left(\mathbb{R}^{s}\right)$. Obviously, this representation of $f$ is closely related to its homogenized version of degree $n$, viz. $H_{n} f=\sum_{\nu \in \Gamma_{s, n}} c_{\nu} B_{\nu}$.

3.3. Dual bases and Euler's identity. The following result generalizes identity (3.1) to the case where the degree of $f$ exceeds $m$. Euler's identity for homogeneous polynomials turns out to be a special case of this result, corresponding to $m=1$.

Proposition 3.4. Let $\mathcal{F}=\left\{f_{\alpha} \mid \alpha \in \Gamma_{s, m}\right\}$ and $\mathcal{G}=\left\{g_{\alpha} \mid \alpha \in \Gamma_{s, m}\right\}$ be a pair of dual bases of $\mathcal{H}_{m}\left(\mathbb{R}^{s}\right)$.

1. Decomposition with respect to dual basis pair: Euler's identity. Every $f \in \mathcal{H}_{n}\left(\mathbb{R}^{s}\right)$, with $0 \leq m \leq n$, can be written as

$$
f=\sum_{\alpha \in \Gamma_{s, m}}\left[f_{\alpha}, f\right] g_{\alpha}
$$

2. Factorization of apolar pairing over a pair of dual bases. For $f \in$ $\mathcal{H}_{n}\left(\mathbb{R}^{s}\right)$ and $g \in \mathcal{H}_{k}\left(\mathbb{R}^{s}\right)$, with $m \leq n \leq k$,

$$
[f, g]=\sum_{\alpha \in \Gamma_{s, m}}\left[\left[f_{\alpha}, f\right],\left[g_{\alpha}, g\right]\right]
$$


In particular, if $m=n$ we have

$$
[f, g]=\sum_{\alpha \in \Gamma_{s, m}}\left[f_{\alpha}, f\right]\left[g_{\alpha}, g\right] .
$$

To see why Property 1 is called Euler's identity, consider the basis $\mathcal{F}=\left\{\left(e_{i}, \cdot\right) \mid\right.$ $i=1, \ldots, s\}$ of $\mathcal{H}_{1}\left(\mathbb{R}^{s}\right)$ (in particular, we take $m=1$ ). Since apolar pairing is a reproducing kernel, we see that $\left[\left(e_{i}, \cdot\right),\left(e_{j}, \cdot\right)\right]=\left(e_{i}, e_{j}\right)=\delta_{i j}$, so the basis $\mathcal{F}$ is its own dual. Furthermore, $\left[\left(e_{i}, \cdot\right), f\right]=\frac{1}{n} \partial_{i} f$ in view of Theorem[2.2, part 2, so in this case the first part of the proposition is just Euler's identity $\sum_{i=1}^{s} x_{i} \partial_{i} f(x)=n f(x)$. In the other extreme case, viz. in case $m=n$, the first property boils down to Marsden's identity if we take $f=(y, \cdot)^{m}$.

Proof of Proposition 3.4. 1. To prove the first part, write $f(y)$, for $y \in \mathbb{R}^{s}$, as the apolar pairing of the homogeneous polynomials $(y, \cdot)^{m}$ and $\left[(y, \cdot)^{n-m}, f\right]$, both belonging to $\mathcal{H}_{m}\left(\mathbb{R}^{s}\right)$. This is justified by first using the fact that apolar pairing is a reproducing kernel to rewrite $f(y)$ as $\left[(y, \cdot)^{n}, f\right]$, and then transposing a factor $(y, \cdot)^{n-m}$ using Theorem 2.2. part 3.

Subsequently express these polynomials with respect to the bases $\mathcal{F}$ and $\mathcal{G}$ of $\mathcal{H}_{m}\left(\mathbb{R}^{s}\right)$, respectively, and compute their apolar pairing using the fact that these bases form a dual pair. In more detail, the expression of the polynomial $(y, \cdot)^{m}$ with respect to the basis $\mathcal{F}$ is equivalent to Marsden's identity:

$$
(y, \cdot)^{m}=\sum_{\alpha \in \Gamma_{s, m}} g_{\alpha}(y) f_{\alpha} .
$$

To write the polynomial $\left[(y, \cdot)^{n-m}, f\right]$ as $\sum_{\alpha} c_{\alpha} g_{\alpha}$, we have to determine $c_{\alpha}=$ $\left[f_{\alpha},\left[(y, \cdot)^{n-m}, f\right]\right]$, cf. (3.1). Using Theorem 2.2 part 3, and the reproducing kernel property, we obtain $c_{\alpha}=\left[f_{\alpha} \cdot(y, \cdot)^{n-m}, f\right]=\left[(y, \cdot)^{n-m},\left[f_{\alpha}, f\right]\right]=$ $\left[f_{\alpha}, f\right](y)$. In other words,

$$
\left[(y, \cdot)^{n-m}, f\right]=\sum_{\alpha \in \Gamma_{s, m}}\left[f_{\alpha}, f\right](y) g_{\alpha} .
$$

Since the bases $\mathcal{F}$ and $\mathcal{G}$ are dual, it follows from (3.4) and (3.5) that

$$
f(y)=\left[(y, \cdot)^{m},\left[(y, \cdot)^{n-m}, f\right]\right]=\sum_{\alpha \in \Gamma_{s, m}}\left[f_{\alpha}, f\right](y) g_{\alpha}(y),
$$

which is just the generalized version of Euler's identity.

2. Use part 1 and the fact that $\left[\left[f_{\alpha}, f\right] g_{\alpha}, g\right]=\left[\left[f_{\alpha}, f\right],\left[g_{\alpha}, g\right]\right]$. In the special case $m=n$, the result follows from the fact that $\left[f_{\alpha}, f\right] \in \mathbb{R}$, so $\left[\left[f_{\alpha}, f\right] g_{\alpha}, g\right]=\left[f_{\alpha}, f\right]\left[g_{\alpha}, g\right]$.

Application: Down-recurrence for Bernstein-Bézier polynomials. As we have seen in Section 3.2 the multivariate Bernstein-Bézier basis is the dual of the lineal basis. Euler's identity can be used to express the Bernstein-Bézier basis functions of degree $n$ in terms of those of degree $m$, for $0 \leq m \leq n$. More precisely, for $\beta \in \Gamma_{s, n}$,

$$
B_{\beta}=\sum_{\alpha \in \Gamma_{s, m}}\left[l_{\alpha}, B_{\beta}\right] B_{\alpha} .
$$


We claim that the polynomial $f=\left[l_{\alpha}, B_{\beta}\right] \in \mathcal{H}_{n-m}\left(\mathbb{R}^{s}\right)$ is a Bernstein-Bézier function:

$$
\left[l_{\alpha}, B_{\beta}\right]= \begin{cases}B_{\beta-\alpha} & \text { if } \alpha \preceq \beta, \\ 0 & \text { if } \alpha \npreceq \beta .\end{cases}
$$

To prove this claim, consider the apolar inner product $\left[l_{\gamma}, f\right]$ for $\gamma \in \Gamma_{s, n-m}$. Since $l_{\alpha} \cdot l_{\gamma}=l_{\alpha+\gamma}$, transposition of a factor $l_{\alpha}$ yields $\left[l_{\gamma}, f\right]=\left[l_{\alpha+\gamma}, B_{\beta}\right]=\delta_{\alpha+\gamma, \beta}$.

First consider the case $\alpha \npreceq \beta$. Then $\alpha+\gamma \neq \beta$, and hence $\left[l_{\gamma}, f\right]=0$, for all $\gamma \in \Gamma_{s, n-m}$. Since $\left\{l_{\gamma} \mid \gamma \in \Gamma_{s, n-m}\right\}$ is a basis of $\mathcal{H}_{n-m}\left(\mathbb{R}^{s}\right)$, it follows that $f=0$ in this case.

If $\alpha \preceq \beta$ the previous derivation shows that $\left[l_{\gamma}, f\right]=\delta_{\gamma, \beta-\alpha}$. Therefore $f=$ $B_{\beta-\alpha}$, since the Bernstein-Bézier basis and the lineal basis of $\mathcal{H}_{n-m}\left(\mathbb{R}^{s}\right)$ form a dual pair.

Plugging the identity (3.7) into (3.6), we obtain the following simple recurrence equation for the multivariate Bernstein-Bézier basis functions $B_{\beta}, \beta \in \Gamma_{s, n}$ :

$$
B_{\beta}=\sum_{\substack{\alpha \in \Gamma_{s, m} \\ \alpha \preceq \beta}} B_{\beta-\alpha} B_{\alpha} .
$$

The well-known one-step recurrence $B_{\beta}=\sum_{i=1}^{s} u_{i} B_{\beta-e_{i}}$, with $B_{\beta-e_{i}}=0$ if $\beta_{i}=0$, is an immediate consequence of (3.8), since $B_{\alpha}=u_{i}$ for $\alpha=e_{i} \in \Gamma_{s, 1}$. See, e.g., [10, Chapter 18]. In the next section we generalize the recurrence (3.8), as well as the identity (3.7), to a rather general class of dual bases.

Application: Degree elevation. Since $\mathcal{P}_{m}\left(\mathbb{R}^{s-1}\right) \subset \mathcal{P}_{n}\left(\mathbb{R}^{s-1}\right)$, for $0 \leq m \leq n$, every polynomial $f \in \mathcal{P}_{m}\left(\mathbb{R}^{s-1}\right)$ can be expressed in the affine Bernstein-Bézier basis of $\mathcal{P}_{n}\left(\mathbb{R}^{s-1}\right)$. The process of expressing the coefficients with respect to the latter basis in terms of those with respect to the former is called degree elevation. Usually the degree is elevated by one, i.e., $n=m+1$. See, e.g., [10, Chapters 5 and 18].

More precisely, consider the barycentric form $f=\sum_{\mu \in \Gamma_{s, m}} c_{\mu} B_{\mu_{1}, \ldots, \mu_{s-1}}^{m}$. In particular, $F=H_{m} f=\sum_{\mu \in \Gamma_{s, m}} c_{\mu} B_{\mu}$. Our goal is to determine the barycentric form of $f$ of degree $n$. In view of identity (3.3), this is equivalent to expressing the polynomial $p^{n-m} F$ with respect to the homogeneous Bernstein-Bézier basis as $p^{n-m} F=\sum_{\nu \in \Gamma_{s, n}} \bar{c}_{\nu} B_{\nu}$.

Since $p=(y, \cdot)$, where $y=e_{1}+\cdots+e_{s}$, Marsden's identity yields

$$
p^{n-m}=\sum_{\gamma \in \Gamma_{s, n-m}} B_{\gamma}(y) l_{\gamma}=\sum_{\gamma \in \Gamma_{s, n-m}}\left(\begin{array}{c}
n-m \\
\gamma
\end{array}\right) l_{\gamma} .
$$

Therefore

$$
\bar{c}_{\beta}=\left[p^{n-m} F, l_{\beta}\right]=\sum_{\gamma \in \Gamma_{s, n-m}}\left(\begin{array}{c}
n-m \\
\gamma
\end{array}\right)\left[l_{\gamma} F, l_{\beta}\right] .
$$

In view of the fact that $l_{\beta}=\left(\begin{array}{c}n \\ \beta\end{array}\right)^{-1} B_{\beta}$, and identity (3.7), we see that

$$
\left[l_{\gamma} F, l_{\beta}\right]=\left[F,\left[l_{\gamma}, l_{\beta}\right]\right]= \begin{cases}\left(\begin{array}{l}
n \\
\beta
\end{array}\right)^{-1}\left[F, B_{\beta-\gamma}\right] & \text { if } \gamma \preceq \beta, \\
0 & \text { if } \gamma \npreceq \beta .\end{cases}
$$


Finally, for $\gamma \preceq \beta$, we have that $\left[F, B_{\beta-\gamma}\right]=\left(\begin{array}{c}n-m \\ \beta-\gamma\end{array}\right)\left[F, l_{\beta-\gamma}\right]=\left(\begin{array}{c}n-m \\ \beta-\gamma\end{array}\right) c_{\beta-\gamma}$, so we get the following degree elevation identity:

$$
\bar{c}_{\beta}=\sum_{\substack{\gamma \in \Gamma_{s, n-m} \\
\gamma \preceq \beta}}\left(\begin{array}{c}
n \\
\beta
\end{array}\right)^{-1}\left(\begin{array}{c}
n-m \\
\gamma
\end{array}\right)\left(\begin{array}{c}
m \\
\beta-\gamma
\end{array}\right) c_{\beta-\gamma}=\left(\begin{array}{c}
n \\
m
\end{array}\right)^{-1} \sum_{\substack{\mu \in \Gamma_{s, m} \\
\mu \preceq \beta}}\left(\begin{array}{c}
\beta \\
\mu
\end{array}\right) c_{\mu} .
$$

Consider the special case where $f$ is the monomial $f(x)=x^{\mu}$, with $x \in \mathbb{R}^{s-1}$ and $\mu \in \Gamma_{s-1, m}$. We apply the previous identity to elevate its degree to $n, n \geq m$. The degree- $m$ barycentric form of $f$ is $\sum_{\lambda \in \Gamma_{s, m}} c_{\lambda} B_{\lambda_{1}, \ldots, \lambda_{s-1}}^{m}$, where $c_{\lambda}=\delta_{\lambda \bar{\mu}}\left(\begin{array}{c}m \\ \mu\end{array}\right)^{-1}$, with $\bar{\mu}=\left(\mu_{1}, \ldots, \mu_{s-1}, 0\right) \in \Gamma_{s, m}$. Hence the coefficients of the barycentric form of degree $n$ are, according to the degree elevation identity above

$$
\bar{c}_{\nu}= \begin{cases}\left(\begin{array}{l}
n \\
m
\end{array}\right)^{-1}\left(\begin{array}{c}
m \\
\mu
\end{array}\right)^{-1}\left(\begin{array}{l}
\nu \\
\bar{\mu}
\end{array}\right) & \text { if } \bar{\mu} \preceq \nu, \\
0 & \text { if } \bar{\mu} \npreceq \nu .\end{cases}
$$

Therefore, the homogenized form of degree $n$ is

$$
H_{n} f=\sum_{\lambda \in \Gamma_{s, n-m}}\left(\begin{array}{c}
n \\
m
\end{array}\right)^{-1}\left(\begin{array}{c}
m \\
\mu
\end{array}\right)^{-1}\left(\begin{array}{c}
\lambda+\bar{\mu} \\
\bar{\mu}
\end{array}\right) B_{\lambda+\bar{\mu}} .
$$

Decomposing $\lambda \in \Gamma_{s, n-m}$ as $\lambda=\kappa+(n-k) e_{s}$, with $\kappa=\kappa_{1} e_{1}+\cdots+\kappa_{s-1} e_{s-1} \in$ $\Gamma_{s-1, k-m}$, we transform the latter identity into

$$
\left(\begin{array}{c}
n \\
m
\end{array}\right)\left(\begin{array}{c}
m \\
\mu
\end{array}\right) H_{n} f=\sum_{k=m}^{n} \sum_{\kappa \in \Gamma_{s-1, k-m}}\left(\begin{array}{c}
\kappa+\mu \\
\mu
\end{array}\right) B_{\kappa+\mu+(n-k) e_{s}} .
$$

Since $B_{\kappa+\mu+(n-k) e_{s}}=H_{n} B_{\kappa+\mu}^{n}$, it follows that, for $x \in \mathbb{R}^{s-1}$ and $\mu \in \Gamma_{s-1, m}$,

$$
\left(\begin{array}{c}
n \\
m
\end{array}\right)\left(\begin{array}{c}
m \\
\mu
\end{array}\right) x^{\mu}=\sum_{k=m}^{n} \sum_{\kappa \in \Gamma_{s-1, k-m}}\left(\begin{array}{c}
\kappa+\mu \\
\mu
\end{array}\right) B_{\kappa+\mu}^{n}(x) .
$$

This identity in fact expresses the monomial basis functions of $\mathcal{P}_{n}\left(\mathbb{R}^{s-1}\right)$ in terms of the affine Bernstein-Bézier functions. In particular, if $s=2$, we have $\Gamma_{s-1, j}=\{j\}$, so the latter identity reduces to the well known relation, cf [10, (5.24)],

$$
\left(\begin{array}{c}
n \\
m
\end{array}\right) x^{m}=\sum_{k=m}^{n}\left(\begin{array}{c}
k \\
m
\end{array}\right) B_{k}^{n}(x) \text {. }
$$

A direct application of Proposition 3.4 yields the following identity for $m$-th derivatives of a homogeneous polynomial of degree $n$ in terms of $(n-m)$-th degree basis functions and the derivatives of the dual basis functions.

Corollary 3.5. Let $\left\{f_{\alpha} \mid \alpha \in \Gamma_{s, n-m}\right\}$ and $\left\{g_{\alpha} \mid \alpha \in \Gamma_{s, n-m}\right\}$ be dual bases of $\mathcal{H}_{n-m}\left(\mathbb{R}^{s}\right)$. Then, for $\xi^{1}, \ldots, \xi^{m} \in \mathbb{R}^{s}$ and $f \in \mathcal{H}_{n}\left(\mathbb{R}^{s}\right)$, with $1 \leq m \leq n$,

$$
D_{\xi^{1}} \cdots D_{\xi^{m}} f=\frac{n !}{(n-m) !} \sum_{\alpha \in \Gamma_{s, n-m}} F_{\alpha}\left(\xi^{1}, \ldots, \xi^{m}\right) g_{\alpha},
$$

where $F_{\alpha}$ is the polar form of $\left[f_{\alpha}, f\right] \in \mathcal{H}_{m}\left(\mathbb{R}^{s}\right)$. In particular, the $m$-th directional derivative with respect to $z \in \mathbb{R}^{s}$ is

$$
D_{z}^{m} f=\frac{n !}{(n-m) !} \sum_{\alpha \in \Gamma_{s, n-m}} f_{\alpha}(z) g_{\alpha}
$$


Proof. Recall from Corollary[2.3, part 1, that

$$
D_{\xi^{1}} \cdots D_{\xi^{m}} f=\frac{n !}{(n-m) !}\left[\left(\xi^{1}, \cdot\right) \cdots\left(\xi^{m}, \cdot\right), f\right] .
$$

Now apply Euler's identity to express the right hand side of the latter identity with respect to the basis $\left\{g_{\alpha} \mid \alpha \in \Gamma_{s, n-m}\right\}$, observing that

$$
\begin{aligned}
{\left[f_{\alpha},\left[\left(\xi^{1}, \cdot\right) \cdots\left(\xi^{m}, \cdot\right), f\right]\right] } & =\left[\left(\xi^{1}, \cdot\right) \cdots\left(\xi^{m}, \cdot\right) f_{\alpha}, f\right] \\
& =\left[\left(\xi^{1}, \cdot\right) \cdots\left(\xi^{m}, \cdot\right),\left[f_{\alpha}, f\right]\right] \\
& =F_{\alpha}\left(\xi^{1}, \ldots, \xi^{m}\right) .
\end{aligned}
$$

Finally, (3.10) follows by taking $\xi^{1}=\ldots=\xi^{m}=z$. This completes the proof.

Application: Derivatives of Bernstein-Bézier polynomials. We continue our leading example by considering derivatives of Bernstein-Bézier basis functions. Take $f=$ $B_{\beta}$, with $\beta \in \Gamma_{s, n}$, in (3.9); then

$$
D_{\xi^{1}} \cdots D_{\xi^{m}} B_{\beta}=\frac{n !}{(n-m) !} \sum_{\substack{\alpha \in \Gamma_{s, n-m} \\ \alpha \preceq \beta}} F_{\beta, \alpha}\left(\xi^{1}, \ldots, \xi^{m}\right) B_{\alpha} .
$$

Here $F_{\beta, \alpha}$ is the polar form of $\left[l_{\alpha}, B_{\beta}\right]$, for $\alpha \in \Gamma_{s, n-m}$. Hence, in view of (3.7),

$$
D_{\xi^{1}} \cdots D_{\xi^{m}} B_{\beta}=\frac{n !}{(n-m) !} \sum_{\substack{\alpha \in \Gamma_{s, n-m} \\ \alpha \preceq \beta}} \mathcal{P}^{m} B_{\beta-\alpha}\left(\xi^{1}, \ldots, \xi^{m}\right) B_{\alpha} .
$$

Passing to the diagonal, i.e., taking $\xi^{1}=\ldots=\xi^{m}=z \in \mathbb{R}^{s}$, we get the well-known expression

$$
D_{z}^{m} B_{\beta}(x)=\frac{n !}{(n-m) !} \sum_{\substack{\alpha \in \Gamma_{s, n-m} \\ \alpha \preceq \beta}} B_{\beta-\alpha}(z) B_{\alpha}(x) .
$$

For $m=1$ this identity reduces to $D_{z} B_{\beta}(x)=n \sum_{i=1}^{s} u_{i}(z) B_{\beta-e_{i}}(x)$, again with the convention that $B_{\beta-e_{i}}=0$ if $\beta_{i}=0$. See also [10, Chapter 18].

\section{Order COMPatible BAses}

In this section we present a rather general framework for constructing computationally convenient polynomial bases. Section 4.1 deals with examples of bases consisting of lineal polynomials, and their dual bases. These dual bases are constructed as generalizations of the Bernstein-Bézier basis, introduced in Section 3.2 We also present an algorithm for converting between representations with respect to two lineal bases. This algorithm is the same as the one presented in Lodha and Goldman [15] (for the trivariate case), but the derivation is different.

Lineal bases are an instance of order compatible bases, a concept introduced in Section 4.2. This general setup is extended in Sections 4.3 and 4.4 where recursive algorithms are derived - more or less routinely - for the computation of these basis functions and their derivatives, and for the evaluation of polynomials expressed in these bases. Among the algorithms derived in this section is a generalization of the classical algorithms of de Boor and de Casteljau for the evaluation of a polynomial. These results are in fact generalizations of the work of Micchelli [17, Section 5.6] and Cavaretta and Micchelli [4]. 


\subsection{Lineal bases.}

4.1.1. Lineal B-basis. As a first example we generalize the lineal basis introduced in Section 3.2 as the dual of the Bernstein-Bézier basis. Every point $x^{i}$ appearing in the basis of $\mathbb{R}^{s}$ in Section 3.2 is replaced with a cloud of points $x^{i, 1}, \ldots, x^{i, n}$. More precisely, we consider an $s \times n$ array $\mathcal{X}$ of points in $\mathbb{R}^{s}$,

$$
\mathcal{X}=\left\{x^{i, j} \mid 1 \leq i \leq s, 1 \leq j \leq n\right\} .
$$

For a multi-index $\alpha \in \Gamma_{s, m}, 1 \leq m \leq n$, we consider the subset $X^{\alpha}$ of $\mathcal{X}$ defined by

$$
X^{\alpha}=\left\{x^{i, j} \in \mathcal{X} \mid 1 \leq j \leq \alpha_{i}, 1 \leq i \leq s\right\} .
$$

(If $\alpha_{i}=0$ the set contains no points of the form $x^{i, j}$.) Note that $X^{\alpha}$ contains $|\alpha|=m$ points, so the lineal polynomial $l_{\alpha}$ defined by

$$
l_{\alpha}(x)=\prod_{u \in X^{\alpha}}(u, x)
$$

is an $s$-variate homogeneous polynomial of degree $m$. By definition, $l_{0}=1$. Note that these lineal polynomials coincide with those of Section 3.2 in case all clouds consist of exactly one point, viz. $x^{i, j}=x^{i}$.

To guarantee that the collection of lineal polynomials $\left\{l_{\alpha} \mid \alpha \in \Gamma_{s, n}\right\}$ forms a basis of $\mathcal{H}_{n}\left(\mathbb{R}^{s}\right)$ we require, roughly speaking, that selecting a point from every cloud yields a basis of $\mathbb{R}^{s}$. More precisely:

Definition 4.1 (Transversal basis property). Array $\mathcal{X}$ has the transversal basis property if, for every multi-index $\alpha=\left(\alpha_{1}, \ldots, \alpha_{s}\right) \in \mathbb{Z}_{\geq 0}^{s}$ with $|\alpha| \leq n-1$ the set

$$
\left\{x^{1, \alpha_{1}+1}, \ldots, x^{s, \alpha_{s}+1}\right\}
$$

is a basis for $\mathbb{R}^{s}$. The corresponding dual basis of $\mathcal{H}_{1}\left(\mathbb{R}^{s}\right)$ is denoted by

$$
\left\{u_{1, \alpha}, \ldots, u_{s, \alpha}\right\} .
$$

The terminology is derived from the observation that the set of points in (4.1) is a transversal of the collection of sets $X_{i}:=\left\{x^{i, j} \mid 1 \leq j \leq n\right\}$, where the index $i$ ranges over $1, \ldots, s$. Note that, by definition, the dual basis satisfies $u_{i, \alpha}\left(x^{j, \alpha_{j}+1}\right)=$ $\delta_{i j}$.

Proposition 4.2. Let the $s \times n$ array $\mathcal{X}$ satisfy the transversal basis property. Then, for $0 \leq m \leq n$ the set $\mathcal{L}_{m}(\mathcal{X})=\left\{l_{\alpha} \mid \alpha \in \Gamma_{s, m}\right\}$ is a basis for $\mathcal{H}_{m}\left(\mathbb{R}^{s}\right)$.

The proof is given in a more general setting in Section 4.2. The basis $\mathcal{L}_{m}(\mathcal{X})$ is called the lineal B-basis of $\mathcal{H}_{m}\left(\mathbb{R}^{s}\right)$ (with respect to the array $\mathcal{X}$ ). Its dual basis, called the $B$-patch basis, is denoted by $\mathcal{B}_{m}(\mathcal{X})$; the basis functions $b_{\mu}, \mu \in \Gamma_{s, m}$, of $\mathcal{B}_{m}(\mathcal{X})$ are called B-patches. These are introduced in Dahmen, Micchelli and Seidel [6] and [17, who use this basis as the starting point for the development of the theory of multivariate B-splines. Some of their basic results are straightforward consequences of the theory developed in the next sections.

The following result gives the decomposition of a polynomial in $\mathcal{H}_{n}\left(\mathbb{R}^{s}\right)$ with respect to the B-patch basis, cf. [17, Proposition 5.4]. 
Corollary 4.3. Let the $s \times n$ array $\mathcal{X}$ satisfy the transversal basis property. Then a polynomial $f \in \mathcal{H}_{n}\left(\mathbb{R}^{s}\right)$ has the following decomposition with respect to the B-patch basis $\mathcal{B}_{n}(\mathcal{X})$ :

$$
f(x)=\sum_{\alpha \in \Gamma_{s, n}} c_{\alpha} b_{\alpha}(x),
$$

where $c_{\alpha}=F\left(X^{\alpha}\right)$, with $F$ the polar form of $f$.

Proof. According to Proposition 3.4.1, the polynomial $f$ has the decomposition (4.2), with $c_{\alpha}=\left[l_{\alpha}, f\right]$. Now Proposition 2.8 yields $c_{\alpha}=F\left(X^{\alpha}\right)$.

The previous result immediately implies that the de Boor-Fix functionals (see Section 3.1), defined with respect to the B-patch basis, are determined by the identity $\lambda_{\alpha}(f)=F\left(X^{\alpha}\right)$, for $\alpha \in \Gamma_{s, n}$. See also [14.

4.1.2. Conversion between lineal B-bases. A central problem in geometric modeling is basis conversion. Here we consider the conversion between representations of a homogeneous polynomial of degree $n$ with respect to two lineal B-bases $\mathcal{L}_{n}(\mathcal{X})$ and $\mathcal{L}_{n}(\overline{\mathcal{X}})$. A similar change of basis algorithm is presented in Lodha and Goldman [15], although their derivation differs from the one presented here in that it is based on duality of so-called simplicial recurrence schemes.

First consider the simplest case in which the $s \times n$ arrays $\mathcal{X}$ and $\overline{\mathcal{X}}$ only differ in their last rows, i.e., $x^{i, j}=\bar{x}^{i, j}$, for $1 \leq i<s$. Both arrays are assumed to satisfy the transversal basis property. Let $h=\sum_{\nu \in \Gamma_{s, n}} c_{\nu} l_{\nu}$ be the representation of a polynomial $h \in \mathcal{H}_{n}\left(\mathbb{R}^{s}\right)$ with respect to the lineal basis $\mathcal{L}_{n}(\mathcal{X})$; then our goal is to compute the representation $h=\sum_{\nu \in \Gamma_{s, n}} \bar{c}_{\nu} \bar{l}_{\nu}$ with respect to the lineal basis $\mathcal{L}_{n}(\overline{\mathcal{X}})$. Isolating linear factors corresponding to the last row of $\mathcal{X}$, we write

$$
h=\sum_{m=0}^{n} g_{m} l_{(n-m) e_{s}},
$$

where $g_{m} \in \mathcal{H}_{m}\left(\mathbb{R}^{s}\right)$ is defined by $g_{m}=\sum_{\substack{\nu \in \Gamma_{s, n} \\ \nu_{s}=n-m}} c_{\nu} l_{\nu-\nu_{s} e_{s}}$. Note that $g_{m}$ has the same representation with respect to both lineal bases, since the lineal polynomials defining $g_{m}$ do not contain linear factors from the last row of $\mathcal{X}$. Therefore

$$
g_{m}=\sum_{\substack{\mu \in \Gamma_{s, m} \\ \mu_{s}=0}} c_{\mu+(n-m) e_{s}} \bar{l}_{\mu} .
$$

For multi-indices $\mu, \nu \in \mathbb{Z}_{\geq 0}^{s}$ with $\mu \preceq \nu$ we observe that $l_{\mu}$ is a divisor of $l_{\nu}$. Their quotient, denoted by $l_{\nu \mid \mu}$, is again a lineal polynomial, viz. the product of all linear factors $(u, \cdot)$ when $u$ ranges over $X^{\nu} \backslash X^{\mu}$. Put

$$
h_{m}=\sum_{k=0}^{m} g_{k} l_{(n-k) e_{s} \mid(n-m) e_{s}} .
$$

Then the following simple properties allow us to set up an iterative basis conversion algorithm:

1. $h_{n}=h$,

2. $h_{0}=c_{n e_{s}}$,

3. $h_{m}=\left(x^{s, n-m+1}, \cdot\right) h_{m-1}+g_{m}$, for $0<m \leq n$. 
Let $a_{\mu}^{m}$ be the coefficients of $h_{m}$ with respect to the basis $\mathcal{L}_{m}(\overline{\mathcal{X}})$, i.e., $h_{m}=$ $\sum_{\mu \in \Gamma_{s, m}} a_{\mu}^{m} \bar{l}_{\mu}$. To transform the third property into a recurrence for these coefficients, we use the fact that $x^{s, n-m+1}=\sum_{i=1}^{s} \bar{u}_{i, \mu}\left(x^{s, n-m+1}\right) \bar{x}^{i, \mu_{i}+1}$, so

$$
\left(x^{s, n-m+1}, \cdot\right)=\sum_{i=1}^{s} \bar{u}_{i, \mu}\left(x^{s, n-m+1}\right) \bar{l}_{\mu+e_{i} \mid \mu} .
$$

The latter identity leads to

$$
\sum_{\mu \in \Gamma_{s, m}} a_{\mu}^{m} \bar{l}_{\mu}=\sum_{\lambda \in \Gamma_{s, m-1}} \sum_{i=1}^{s} \bar{u}_{i, \lambda}\left(x^{s, n-m+1}\right) a_{\lambda}^{m-1} \bar{l}_{\lambda+e_{i}}+\sum_{\substack{\mu \in \Gamma_{s, m} \\ \mu_{s}=0}} c_{\mu+(n-m) e_{s}} \bar{l}_{\mu},
$$

for $m>0$ and $\mu \in \Gamma_{s, m}$. Collecting coefficients corresponding to the same basis functions, we finally obtain the following recurrence:

$$
\left\{\begin{aligned}
a_{0}^{0}= & c_{n e_{s}}, \\
a_{\mu}^{m}=\sum_{i=1}^{s} \bar{u}_{i, \mu-e_{i}}\left(x^{s, n-m+1}\right) a_{\mu-e_{i}}^{m-1}+\delta_{0, \mu_{s}} c_{\mu+(n-m) e_{s}}, & \text { if } m>0, \mu \in \Gamma_{s, m} .
\end{aligned}\right.
$$

Summarizing, recurrence (4.3) yields an algorithm that converts the representation $h=\sum_{\nu} c_{\nu} l_{\nu}$ with respect to the lineal B-basis $\mathcal{L}_{n}(\mathcal{X})$ to the representation $h=$ $\sum_{\nu} a_{\nu}^{n} \bar{l}_{\nu}$ with respect to the the lineal B-basis $\mathcal{L}_{n}(\overline{\mathcal{X}})$.

To deal with the general case, let $\mathcal{X}_{k}, 0 \leq k \leq s$, be the $s \times n$ array obtained from $\mathcal{X}$ by replacing the last $k$ columns with the corresponding columns of $\overline{\mathcal{X}}$. Obviously $\mathcal{X}_{0}=\mathcal{X}, \mathcal{X}_{s}=\overline{\mathcal{X}}$, and $\mathcal{X}_{k}$ and $\mathcal{X}_{k+1}$ differ only in their $k$-th column. Under the assumption that $\mathcal{X}_{k}$ satisfies the transversal basis property, we may run the algorithm just derived to convert a representation with respect to $\mathcal{L}_{n}\left(\mathcal{X}_{k}\right)$ into the representation with respect to $\mathcal{L}_{n}\left(\mathcal{X}_{k+1}\right)$, successively for $k=0, \ldots, s-1$. This is exactly the approach of Lodha and Goldman [15].

Application: Conversion from monomial to Bernstein-Bézier form. As a simple application we consider the conversion of a univariate polynomial of the form $h(t)=$ $\sum_{k=0}^{n} a_{k} t^{k}$ to its Bernstein-Bézier form $h(t)=\sum_{k=0}^{n} b_{k} B_{k}^{n}(t)$. To prepare for the application of the recurrence (4.3) we first homogenize as in Section 3.2. In other words, we use the fact that $h(t)=H(t, 1-t)$, where the homogeneous polynomial $H$ is defined by $H\left(x_{1}, x_{2}\right)=\sum_{k=0}^{n} c_{(k, n-k)} x_{1}^{k}\left(x_{1}+x_{2}\right)^{n-k}$, with $c_{(k, n-k)}=a_{k}$. After conversion to the monomial form $H\left(x_{1}, x_{2}\right)=\sum_{k=0}^{n} \bar{c}_{(k, n-k)} x_{1}^{k} x_{2}^{n-k}$, we see that the Bernstein-Bézier coefficients are $b_{k}=\bar{c}_{(k, n-k)} /\left(\begin{array}{l}n \\ k\end{array}\right)$.

The actual conversion is established by applying the recurrence (4.3) in case $s=2$, with $x^{1, j}=e_{1}, x^{2, j}=e_{1}+e_{2}, \bar{x}^{1, j}=e_{1}$ and $\bar{x}^{2, j}=e_{2}$. Note that in this case $\bar{u}_{i, \mu}\left(x^{2, j}\right)=1$, for $i=1,2$ and $\mu \in \Gamma_{s, m}$ with $0 \leq m<n$. In the notation of (4.3) we put $c_{k}^{m}=a_{(k, m-k)}^{m}$, for $0 \leq m \leq n$ and $0 \leq k \leq m$. This yields the recurrence

$$
\left\{\begin{array}{l}
c_{0}^{0}=c_{n}, \\
c_{k}^{m}=c_{k}^{m-1}+c_{k-1}^{m-1}+\delta_{k m} c_{m}, \text { if } m>0 \text { and } 0 \leq k \leq m .
\end{array}\right.
$$

To obtain the coefficients of the Bernstein-Bézier form, let $b_{k}^{m}=c_{k}^{m} /\left(\begin{array}{c}m \\ k\end{array}\right)$; then (4.4) transforms into

$$
\left\{\begin{array}{l}
b_{0}^{0}=c_{n}, \\
b_{k}^{m}=\left(1-\frac{k}{m}\right) b_{k}^{m-1}+\frac{k}{m} b_{k-1}^{m-1}+\delta_{k m} c_{m}, \text { if } m>0 \text { and } 0 \leq k \leq m .
\end{array}\right.
$$


The previous recurrence translates directly into the following in-situ algorithm:

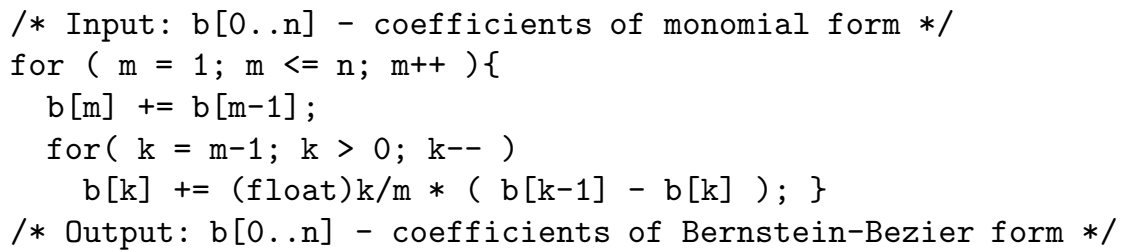

4.1.3. Lineal knot basis. In the second example of a lineal basis of $\mathcal{H}_{n}\left(\mathbb{R}^{s}\right)$ the points defining the lineal polynomials are taken from a (multi-)set $X$, consisting of $n+s-1$ points in $\mathbb{R}^{s}$. The index set $\Lambda_{s, n}$ is the collection of all $n$-element subsets of $\{1, \ldots, n+s-1\}$, which has cardinality equal to $\operatorname{dim} \mathcal{H}_{n}\left(\mathbb{R}^{s}\right)$. With an index $I \in \Lambda_{s, n}$ we associate the multi-set $X^{I}=\left\{x_{i} \mid i \in I\right\}$, and the lineal polynomial $l_{I} \in \mathcal{H}_{n}\left(\mathbb{R}^{s}\right)$ defined by

$$
l_{I}(x)=\prod_{u \in X^{I}}(u, x) .
$$

Again $l_{\emptyset}=1$, by definition. We say that $X$ is in general position if every subset of $X$ consisting of $s$ points is a basis of $\mathbb{R}^{s}$.

Proposition 4.4. Let the set $X$ be in general position. Then

$$
\mathcal{L}_{m}(X)=\left\{l_{I} \mid I \in \Lambda_{s, m}\right\}
$$

is a basis for $\mathcal{H}_{m}\left(\mathbb{R}^{s}\right)$, for $0 \leq m \leq n$.

The proof of this result is also deferred to Section 4.2 In the theory of multidimensional simplex splines points in $X$ are called knots; see e.g. 17 and 18. This is why we call $\mathcal{L}_{m}(X)$ the lineal knot basis of $\mathcal{H}_{m}\left(\mathbb{R}^{s}\right)$ (associated with the knot set $X)$. In analogy with the B-patches, the dual basis functions are called knot-patches; they are denoted by $N_{I}, I \in \Lambda_{s, m}$, with the convention $\left[l_{I}, N_{J}\right]=\delta_{I J}$.

An immediate application of this proposition is the polar interpolation property; see Neamtu [18].

Corollary 4.5. Let $X$ be a set of $n+s-1$ points in $\mathbb{R}^{s}$ in general position. Then there is a unique polynomial $f \in \mathcal{H}_{n}\left(\mathbb{R}^{s}\right)$ whose polar form $F$ attains prescribed values at all subsets of $X$ of cardinality $n$. More precisely, given $c_{I} \in \mathbb{R}^{s}$ for all $I \in \Lambda_{s, n}$, the polar form $F$ satisfies $F\left(X^{I}\right)=c_{I}$, for $I \in \Lambda_{s, n}$.

Proof. In view of Proposition 4.4, the collection of lineal polynomials $l_{I} \in \mathcal{H}_{n}\left(\mathbb{R}^{s}\right)$, where $I$ ranges over $\Lambda_{s, n}$, is a basis of $\mathcal{H}_{n}\left(\mathbb{R}^{s}\right)$. Then $f=\sum_{I \in \Lambda_{s, n}} c_{I} N_{I}$ is a polynomial in $\mathcal{H}_{n}\left(\mathbb{R}^{s}\right)$, whose polar form satisfies $\mathcal{P}^{n} f\left(X^{I}\right)=\left[l_{I}, f\right]$; see Proposition 2.8 It follows from Euler's identity, cf. Proposition 3.4 part 1 , that $\left[l_{I}, f\right]=c_{I}$, so the proof is complete.

4.2. Order compatible bases. We now study more general collections $\left\{f_{\nu} \mid \nu \in\right.$ $\left.\mathcal{I}_{s, n}\right\}$ of polynomials of degree $n$, and in particular deal with the problem of determining whether this collection is a basis for $\mathcal{H}_{n}\left(\mathbb{R}^{s}\right)$. Here $\mathcal{I}_{s, n}, n \geq 0$, are general index sets such that

$$
\# \mathcal{I}_{s, n}=\operatorname{dim} \mathcal{H}_{n}\left(\mathbb{R}^{s}\right)=\left(\begin{array}{c}
n+s-1 \\
n
\end{array}\right),
$$


and such that $\mathcal{I}_{s, m}$ and $\mathcal{I}_{s, n}$ are disjoint whenever $m$ and $n$ are distinct non-negative integers. The union of all index sets $\mathcal{I}_{s, n}, n \geq 0$, is denoted by $\mathcal{I}_{s}$. For convenience, we denote the single element of $\mathcal{I}_{s, 0}$ by 0 . Obviously, the set of multi-indices $\Gamma_{s, n}$ of weight $n$ is an example of such an index set.

A partial order $\preceq$ on $\mathcal{I}_{s}$ is called regular if, for $0 \leq m \leq n$ and $\mu \in \mathcal{I}_{s, m}$, the set $\mathcal{I}_{s, n-m}(\mu)$, defined by

$$
\mathcal{I}_{s, n-m}(\mu)=\left\{\nu \mid \nu \in \mathcal{I}_{s, n} \text { and } \mu \preceq \nu\right\},
$$

consists of $\left(\begin{array}{c}n-m+s-1 \\ n-m\end{array}\right)=\operatorname{dim} \mathcal{H}_{n-m}\left(\mathbb{R}^{s}\right)$ elements. Throughout this section we assume that $\mathcal{I}_{s}$ is an index set endowed with a regular partial order $\preceq$.

Consider collections $\mathcal{F}_{m}=\left\{f_{\mu} \mid \mu \in \mathcal{I}_{s, m}\right\}$ and $\mathcal{F}_{n}=\left\{f_{\nu} \mid \nu \in \mathcal{I}_{s, n}\right\}$ of homogeneous polynomials on $\mathbb{R}^{s}$ of degree $m$ and $n$, respectively. We say that the pair $\left(\mathcal{F}_{m}, \mathcal{F}_{n}\right)$, with $0 \leq m \leq n$, is $\preceq$-compatible if

1. $0 \notin \mathcal{F}_{m}$ and $0 \notin \mathcal{F}_{n}$.

2. For $\mu \in \mathcal{I}_{s, m}$ and $\nu \in \mathcal{I}_{s, n}$, with $\mu \preceq \nu$, the polynomial $f_{\mu}$ is a divisor of $f_{\nu}$. The quotient $f_{\nu} / f_{\mu}$ is denoted by $f_{\nu \mid \mu}$. Obviously $f_{\nu \mid \mu} \in \mathcal{H}_{n-m}\left(\mathbb{R}^{s}\right)$.

For convenience, we assume that $\mathcal{F}_{0}$ consists of the single constant polynomial 1. Under this assumption $f_{\mu \mid 0}=f_{\mu}$.

The sets of lineal polynomials, introduced in Section 4.1, are easily seen to be order compatible. More precisely, consider the lineal B-bases. The partial order $\preceq$ on the set of multi-indices $\mathbb{Z}_{\geq 0}^{s}$ is regular, since the set $\Gamma_{s, n}(\alpha)$ of successors of $\alpha \in$ $\Gamma_{s, m}$ in $\Gamma_{s, n}$ consists of all multi-indices of the form $\alpha+\gamma$, where $\gamma$ ranges over the set $\Gamma_{s, n-m}$. In particular, this set contains $\operatorname{dim} \mathcal{H}_{n-m}\left(\mathbb{R}^{s}\right)$ elements. Furthermore, if $\alpha \preceq \beta$, then $X^{\alpha} \subset X^{\beta}$, and hence the lineal polynomial $l_{\alpha}$ is a divisor of $l_{\beta}$. In other words, $\left(\mathcal{L}_{m}(\mathcal{X}), \mathcal{L}_{n}(\mathcal{X})\right)$ is an order compatible pair, for $0 \leq m \leq n$.

With regard to the lineal knot bases we consider the partial order $\preceq$ on the index set $\Lambda_{s}=\bigcup_{n \geq 0} \Lambda_{s, n}$, defined by $I \preceq J$ if $I \subset J$, for $I, J \in \Lambda_{s}$. For a fixed $I \in \Lambda_{s, m}$, the set $\{1, \ldots, n+s-1\} \backslash I$ consists of $n-m+s-1$ elements, so there are $\left(\begin{array}{c}n-m+s-1 \\ n-m\end{array}\right)=\operatorname{dim} \mathcal{H}_{n-m}\left(\mathbb{R}^{s}\right)$ distinct ways to extend $I$ to an $n$-element subset of $\{1, \ldots, n+s-1\}$. In other words: the set $\left\{J \in \Lambda_{s, n} \mid I \preceq J\right\}$ contains $\operatorname{dim} \mathcal{H}_{n-m}\left(\mathbb{R}^{s}\right)$ elements; hence $\preceq$ is a regular partial order. Furthermore, consider $I \in \Lambda_{s, m}$ and $J \in \Lambda_{s, n}, 0 \leq m \leq n$, with $I \preceq J$. Then $X^{I} \subset X^{J}$; hence $l_{I}$ is a divisor of $l_{J}$. So also in this case the pair $\left(\mathcal{L}_{m}(X), \mathcal{L}_{n}(X)\right)$ is order compatible whenever $0 \leq m \leq n$.

The following result is the crucial tool for deciding when a collection of polynomials is a basis for $\mathcal{H}_{n}\left(\mathbb{R}^{s}\right)$.

Theorem 4.6. Let $0 \leq m \leq n$, and let the pair $\left(\mathcal{F}_{m}, \mathcal{F}_{n}\right)$ be $\preceq$-compatible.

1. If $\mathcal{F}_{m}$ be a basis of $\mathcal{H}_{m}\left(\mathbb{R}^{s}\right)$, and for all $\mu \in \mathcal{I}_{s, m}$ the set

$$
\mathcal{F}_{n-m}(\mu):=\left\{f_{\nu \mid \mu} \mid \nu \in \mathcal{I}_{s, n} \text { and } \mu \preceq \nu\right\}
$$

is a basis of $\mathcal{H}_{n-m}\left(\mathbb{R}^{s}\right)$, then $\mathcal{F}_{n}$ is a basis of $\mathcal{H}_{n}\left(\mathbb{R}^{s}\right)$.

2. If $\mathcal{F}_{n}$ is a basis of $\mathcal{H}_{n}\left(\mathbb{R}^{s}\right)$, then $\mathcal{F}_{n-m}(\mu)$ is a basis of $\mathcal{H}_{n-m}\left(\mathbb{R}^{s}\right)$, for all $\mu \in \mathcal{I}_{s, m}$.

The reader may wonder whether, under the assumptions of part 2 , the set $\mathcal{F}_{m}$ is also a basis of $\mathcal{H}_{m}\left(\mathbb{R}^{s}\right)$. This turns out to be true under a mild additional assumption on the index set $\mathcal{I}_{s}$. This assumption turns out to hold for the index sets $\mathbb{Z}_{\geq 0}^{s}$ and $\Lambda_{s}$, introduced in Section 4.1. We don't need this stronger version 
of Theorem 4.6] in this paper, so the details, which are not completely trivial, are omitted from this paper.

Before proceeding to the proof, let us show how to apply this theorem to the collections of lineal polynomials in Section 4.1.

Proof of Proposition 4.2. The claim is obviously true for $m=0$, since $\mathcal{L}_{0}(\mathcal{X})=\{1\}$ is a basis for $\mathcal{H}_{0}\left(\mathbb{R}^{s}\right)$. So assume the claim is proved for $0 \leq m<n$. Then, for $\alpha \in \Gamma_{s, m}$,

$$
\mathcal{F}_{1}(\alpha)=\left\{l_{\alpha+e_{1} \mid \alpha}, \ldots, l_{\alpha+e_{s} \mid \alpha}\right\}=\left\{\left(x^{1, \alpha_{1}+1}, \cdot\right), \ldots,\left(x^{s, \alpha_{s}+1}, \cdot\right)\right\},
$$

since $\mathcal{I}_{s, 1}(\alpha)=\Gamma_{s, 1}(\alpha)=\left\{\alpha+e_{1}, \ldots, \alpha+e_{s}\right\}$. Therefore the transversal property holds iff $\mathcal{F}_{1}(\alpha)$ is a basis of $\mathcal{H}_{1}\left(\mathbb{R}^{s}\right)$, for $\alpha \in \mathcal{H}_{m}\left(\mathbb{R}^{s}\right)$. Using Theorem 4.6 we conclude that $\mathcal{L}_{m+1}(\mathcal{X})$ is a basis of $\mathcal{H}_{m+1}\left(\mathbb{R}^{s}\right)$. Proceeding inductively, we conclude that $\mathcal{L}_{m}(\mathcal{X})$ is a basis of $\mathcal{H}_{m}\left(\mathbb{R}^{s}\right)$ for $0 \leq m \leq n$.

Proof of Proposition 4.4. We only have to observe that for an index $I \in \Lambda_{s, m}$, i.e., a subset of $X$ of cardinality $m$, with $0 \leq m<n$, the set $\mathcal{I}_{s, 1}(I)=\Lambda_{s, 1}(I)$ consists of the $m+1$-element subsets of $\left\{x_{1}, \ldots, x_{m+s}\right\}$ containing $I$. Since $X$ is in general position, its $s$-element subset $\left\{x_{j} \mid j \in\{1, \ldots, m+s\}-I\right\}$ is a basis of $\mathbb{R}^{s}$, and hence $\mathcal{F}_{1}(I)=\left\{\left(x_{j}, \cdot\right) \mid j \in\{1, \ldots, m+s\}-I\right\}$ is a basis for $\mathcal{H}_{1}\left(\mathbb{R}^{s}\right)$. This observation allows us to set up an inductive argument similar to the proof of Proposition 4.2 .

Proof of Theorem 4.6. 1. First observe that the cardinality of $\mathcal{F}_{n}$ is equal to the dimension of $\mathcal{H}_{n}\left(\mathbb{R}^{s}\right)$, so it is sufficient to prove that the elements of $\mathcal{F}_{n}$ are linearly independent. So let $f \in \mathcal{H}_{n}\left(\mathbb{R}^{s}\right)$, and assume that $\left[f_{\nu}, f\right]=0$ for all $\nu \in \mathcal{I}_{s, n}$. Then all we have to prove is $f=0$.

To this end fix $\mu \in \mathcal{I}_{s, m}$, and let $\left\{g_{\nu \mid \mu} \mid \nu \in \mathcal{I}_{s, n-m}(\mu)\right\}$ be the dual basis of $\mathcal{F}_{n-m}(\mu)=\left\{f_{\nu \mid \mu} \mid \nu \in \mathcal{I}_{s, n-m}(\mu)\right\}$. Using Proposition [3.4, part 1, to express $\left[f_{\mu}, f\right] \in \mathcal{H}_{n-m}\left(\mathbb{R}^{s}\right)$ with respect to this dual basis, we get

$$
\left[f_{\mu}, f\right]=\sum_{\nu \in \mathcal{I}_{s, n-m}(\mu)}\left[f_{\nu \mid \mu},\left[f_{\mu}, f\right]\right] g_{\nu \mid \mu}=0,
$$

since $\left[f_{\nu \mid \mu},\left[f_{\mu}, f\right]\right]=\left[f_{\nu \mid \mu} \cdot f_{\mu}, f\right]=\left[f_{\nu}, f\right]=0$. Therefore $\left[f_{\mu}, f\right]=0$, for all $\mu \in \mathcal{I}_{s, m}$, so $f=0$ according to Lemma 2.5.

2. Since the partial order $\preceq$ is regular, $\mathcal{F}_{n-m}(\mu)$ contains $\operatorname{dim} \mathcal{H}_{n-m}\left(\mathbb{R}^{s}\right)$ elements. Hence it suffices to prove that $\mathcal{F}_{n-m}(\mu)$ is a linearly independent set. So let $c_{\nu}$ be real constants such that $\sum_{\nu \in \mathcal{I}_{s, n-m}(\mu)} c_{\nu} f_{\nu \mid \mu}=0$. Since $f_{\nu \mid \mu} \cdot f_{\mu}=f_{\nu}$, multiplying both sides of the latter equality by $f_{\mu}$ shows that $\sum_{\nu \in \mathcal{I}_{s, n-m}(\mu)} c_{\nu} f_{\nu}=0$. In view of the fact that $\left\{f_{\nu} \mid \nu \in \mathcal{I}_{s, n-m}(\mu)\right\}$ is a subset of the basis $\mathcal{F}_{n}$, it follows that $c_{\nu}=0$ for $\nu \in \mathcal{I}_{s, n-m}(\mu)$. In other words, the collection $\mathcal{F}_{n-m}(\mu)$ is linearly independent.

Under the hypothesis of Theorem 4.6, 2 the elements of the dual basis of $\mathcal{F}_{n-m}(\mu)$ can be given explicitly, as reflected by the following result, cf. (3.7):

Proposition 4.7. Let $0 \leq m \leq n$, and let $\left(\mathcal{F}_{m}, \mathcal{F}_{n}\right)$ be an order compatible pair such that $\mathcal{F}_{n}=\left\{f_{\nu} \mid \nu \in \mathcal{I}_{s, n}\right\}$ is a basis of $\mathcal{H}_{n}\left(\mathbb{R}^{s}\right)$, with dual basis $\mathcal{G}_{n}=\left\{g_{\nu} \mid \nu \in\right.$ $\left.\mathcal{I}_{s, n}\right\}$. Let $\mu \in \mathcal{I}_{s, m}$.

1. The dual basis of $\mathcal{F}_{n-m}(\mu)$ is $\mathcal{G}_{n-m}(\mu)=\left\{g_{\nu \mid \mu} \mid \nu \in \mathcal{I}_{s, n}\right.$ and $\left.\mu \preceq \nu\right\}$, where

$$
g_{\nu \mid \mu}=\left[f_{\mu}, g_{\nu}\right] \in \mathcal{H}_{n-m}\left(\mathbb{R}^{s}\right) .
$$


2. For $\nu \in \mathcal{I}_{s, n}$, with $\mu \npreceq \nu$,

$$
\left[f_{\mu}, g_{\nu}\right]=0 \in \mathcal{H}_{n-m}\left(\mathbb{R}^{s}\right) .
$$

Proof. 1. This follows from the fact that, for $\lambda \in \mathcal{I}_{s, n}$ with $\mu \preceq \lambda$,

$$
\left[f_{\lambda \mid \mu}, g_{\nu \mid \mu}\right]=\left[f_{\lambda \mid \mu},\left[f_{\mu}, g_{\nu}\right]\right]=\left[f_{\lambda \mid \mu} f_{\mu}, g_{\nu}\right]=\left[f_{\lambda}, g_{\nu}\right]=\delta_{\lambda \nu}
$$

2. Let $g=\left[f_{\mu}, g_{\nu}\right] \in \mathcal{H}_{n-m}\left(\mathbb{R}^{s}\right)$. We show that $[f, g]=0$, for all $f \in$ $\mathcal{H}_{n-m}\left(\mathbb{R}^{s}\right)$. The proof of the first part shows that, for $\lambda \in \mathcal{I}_{s, n}$ with $\mu \preceq \lambda$,

$$
\left[f_{\lambda \mid \mu}, g\right]=0,
$$

since $\lambda \npreceq \nu$ (otherwise $\mu \preceq \nu$ ). According to Theorem 4.6]2, the set $\left\{f_{\lambda \mid \mu} \mid \lambda \in\right.$ $\mathcal{I}_{s, n-m}$ and $\left.\mu \preceq \lambda\right\}$ is a basis of $\mathcal{H}_{n-m}\left(\mathbb{R}^{s}\right)$, so we even have $[f, g]=0$, for all $f \in \mathcal{H}_{n-m}\left(\mathbb{R}^{s}\right)$. Now apply Lemma 2.5 to conclude that $g=0$.

The dual basis $\mathcal{G}_{n-m}(\mu)$ can be used to express the $m$-th derivative of a polynomial $h \in \mathcal{H}_{n}\left(\mathbb{R}^{s}\right)$ with respect to the basis $\mathcal{G}_{n-m}$ :

Proposition 4.8. Let $0 \leq m \leq n$, and let $\left(\mathcal{F}_{n-m}, \mathcal{F}_{n}\right)$ be an order compatible pair such that $\mathcal{F}_{n}=\left\{f_{\nu} \mid \nu \in \mathcal{I}_{s, n}\right\}$ is a basis of $\mathcal{H}_{n}\left(\mathbb{R}^{s}\right)$, with dual basis $\mathcal{G}_{n}=\left\{g_{\nu} \mid\right.$ $\left.\nu \in \mathcal{I}_{s, n}\right\}$. The $m$-th derivative of $h=\sum_{\nu \in \mathcal{I}_{s, n}} c_{\nu} g_{\nu} \in \mathcal{H}_{n}\left(\mathbb{R}^{s}\right)$ with respect to $\xi^{1}, \ldots, \xi^{m} \in \mathbb{R}^{s}$ has the decomposition

$$
D_{\xi^{1}} \cdots D_{\xi^{m}} h(x)=\frac{n !}{(n-m) !} \sum_{\mu \in \mathcal{I}_{s, n-m}} \sum_{\substack{\nu \in \mathcal{I}_{s, n} \\ \nu \succeq \mu}} c_{\nu} G_{\nu \mid \mu}\left(\xi^{1}, \ldots, \xi^{m}\right) g_{\mu}(x),
$$

with respect to the basis $\mathcal{G}_{n-m}$ of $\mathcal{H}_{n-m}\left(\mathbb{R}^{s}\right)$, where $G_{\nu \mid \mu}$ is the polar form of $g_{\nu \mid \mu}=$ $\left[f_{\mu}, g_{\nu}\right] \in \mathcal{H}_{m}\left(\mathbb{R}^{s}\right)$. In particular, for $z \in \mathbb{R}^{s}$

$$
D_{z}^{m} h(x)=\frac{n !}{(n-m) !} \sum_{\mu \in \mathcal{I}_{s, n-m}} \sum_{\substack{\nu \in \mathcal{I}_{s, n} \\ \nu \succeq \mu}} c_{\nu} g_{\nu \mid \mu}(u) g_{\mu}(x) .
$$

Proof. Applying Corollary 3.5. we see that

$$
D_{\xi^{1}} \cdots D_{\xi^{m}} h(x)=\frac{n !}{(n-m) !} \sum_{\mu \in \mathcal{I}_{s, n-m}} C_{\mu}^{m}\left(\xi^{1}, \ldots, \xi^{m}\right) g_{\mu}(x),
$$

where $C_{\mu}^{m}$ is the polar form of $\left[f_{\mu}, h\right] \in \mathcal{H}_{m}\left(\mathbb{R}^{s}\right)$. In view of Corollary 2.3, part 1, we have, with $l=\left(\xi^{1}, \cdot\right) \cdots\left(\xi^{m}, \cdot\right)$,

$$
C_{\mu}^{m}\left(\xi^{1}, \ldots, \xi^{m}\right)=\sum_{\nu \in \mathcal{I}_{s, n}} c_{\nu}\left[l,\left[f_{\mu}, g_{\nu}\right]\right]=\sum_{\substack{\nu \in \mathcal{I}_{s, n} \\ \nu \succeq \mu}} c_{\nu}\left[l, g_{\nu \mid \mu}\right],
$$

so the proof is complete, since $\left[l, g_{\nu \mid \mu}\right]=G_{\nu \mid \mu}\left(\xi^{1}, \ldots, \xi^{m}\right)$.

Derivatives of a polynomial in Bézier form. Consider the Bernstein-Bézier basis of $\mathcal{H}_{n}\left(\mathbb{R}^{s}\right)$, indexed by the set of multi-indices $\Gamma_{s, n}$. More precisely, for $\lambda \in \Gamma_{s, m}$, let $f_{\lambda}(x)=x^{\lambda}=l_{\lambda}(x)$; then, as we have seen in Section 3.2, the dual basis functions are the homogeneous Bernstein-Bézier polynomials, i.e., $g_{\lambda}=B_{\lambda}$. For $\lambda \in \Gamma_{s, m}$ the dual of the relative basis $\mathcal{F}_{n-m}(\lambda)$ consists of the functions $g_{\mu \mid \lambda}=\left[l_{\lambda}, B_{\mu}\right]=B_{\mu-\lambda}$, 
where $\mu \in \Gamma_{s, n}$ with $\mu \succeq \lambda$. Therefore, if $h=\sum_{\nu \in \Gamma_{s, n}} c_{\nu} B_{\nu}$, identity (4.5) reduces to

$$
D_{z}^{m} h(x)=\frac{n !}{(n-m) !} \sum_{\lambda \in \Gamma_{s, n-m}} \sum_{\gamma \in \Gamma_{s, m}} c_{\gamma+\lambda} B_{\gamma}(z) B_{\lambda}(x) .
$$

Derivatives of a Bézier curve. Consider a not necessarily homogeneous polynomial $f(t)$ of degree $n$ in $t \in \mathbb{R}$ with Bézier form $f(t)=\sum_{k=0}^{n} c_{k} B_{k}^{n}(t)$. Since $B_{k}^{n}(t)=$ $\left.B_{(k, n-k)}(t, 1-t)\right)$, we see that $f(t)=F(t, 1-t)$, where $F \in \mathcal{H}_{n}\left(\mathbb{R}^{2}\right)$ is defined by $F(x)=\sum_{k=0}^{n} c_{k} B_{(k, n-k)}(x)$. We can express the derivatives of $f(t)$ in terms of its barycentric form $F$ as follows: $f^{(m)}(t)=D_{z}^{m} F(t, 1-t)$, where $z=(1,-1) \in \mathbb{R}^{2}$. Applying (4.7), we get

$$
D_{z}^{m} F(x)=\frac{n !}{(n-m) !} \sum_{k=0}^{n-m} \sum_{l=0}^{m} c_{k+l} B_{l}^{m}(z) B_{k}^{n-m}(x) .
$$

Since, by definition,

$$
\sum_{l=0}^{m} c_{k+l} B_{l}^{m}(z)=\sum_{l=0}^{m}\left(\begin{array}{c}
m \\
l
\end{array}\right)(-1)^{m-l} c_{k+l}=\triangle^{m} c_{k},
$$

the $m$-th divided difference of the sequence $c_{k}, c_{k+1}, \ldots, c_{k+m}$, we conclude that

$$
f^{(m)}(t)=\frac{n !}{(n-m) !} \sum_{k=0}^{n-m} \triangle^{m} c_{k} B_{k}^{n-m}(t) .
$$

This is Farin [10, (4.27)].

4.3. Downward recursion schemes. In the sequel we consider a family of bases $\mathcal{F}_{n}$ of $\mathcal{H}_{n}\left(\mathbb{R}^{s}\right), n \geq 0$, such that $\mathcal{F}_{0}=\{1\}$, and for every $n \geq 0$ the pair $\left(\mathcal{F}_{n}, \mathcal{F}_{n+1}\right)$ is order compatible (with respect to some fixed regular partial order on a generalized index set $\mathcal{I}_{s}$; see Section 4.2). We adopt the same notation as in the latter section. In particular, $\mathcal{G}_{n}$ denotes the dual basis of $\mathcal{F}_{n}$. Furthermore, the polynomials $g_{\nu \mid \mu}=\left[f_{\mu}, g_{\nu}\right]$, with $\nu$ ranging over all indices in $\mathcal{I}_{s, n}$ with $\mu \preceq \nu$, form the dual basis of $\mathcal{F}_{n-m}(\mu)$, consisting of the polynomials $f_{\nu \mid \mu}$, with $\nu$ ranging over all indices in $\mathcal{I}_{s, n}$ with $\mu \preceq \nu$ (see also Proposition 4.7).

The following rather general result will allow us to derive several useful recurrence relations for basis polynomials.

Proposition 4.9. With the previous assumptions, let $0 \leq m \leq k \leq n$, and let $\mu \in \mathcal{I}_{s, m}$ and $\nu \in \mathcal{I}_{s, n}$ with $\mu \preceq \nu$. Then, for $f \in \mathcal{H}_{l}\left(\mathbb{R}^{s}\right)$, with $l$ such that $k+l \leq n$,

$$
\left[f, g_{\nu \mid \mu}\right]=\sum_{\lambda}\left[f, g_{\nu \mid \lambda}\right] g_{\lambda \mid \mu}
$$

where the summation ranges over all indices $\lambda \in \mathcal{I}_{s, k}$ with $\mu \preceq \lambda \preceq \nu$.

Proof. Writing $h=\left[f, g_{\nu \mid \mu}\right] \in \mathcal{H}_{n-m-l}\left(\mathbb{R}^{s}\right)$, and observing that $k-m \leq n-m-l$, we may apply Proposition [3.4. part 1, to decompose $h$ with respect to any dual 
basis pair of $\mathcal{H}_{k-m}\left(\mathbb{R}^{s}\right)$. In particular we consider the dual bases $\left\{f_{\lambda \mid \mu}\right\}$ and $\left\{g_{\lambda \mid \mu}\right\}$, where $\lambda$ ranges over all indices in $\mathcal{I}_{s, k}$ with $\mu \preceq \lambda$. Hence

$$
h=\sum_{\lambda \in \mathcal{I}_{s, k} \text { and } \mu \preceq \lambda}\left[f_{\lambda \mid \mu}, h\right] g_{\lambda \mid \mu} .
$$

Since $(k-m)+l \leq n-m$, we repeatedly apply Theorem 2.2 , part 3 , to transpose homogeneous factors:

$$
\begin{aligned}
{\left[f_{\lambda \mid \mu}, h\right]=\left[f_{\lambda \mid \mu},\left[f, g_{\nu \mid \mu}\right]\right] } & =\left[f_{\lambda \mid \mu} \cdot f, g_{\nu \mid \mu}\right] \\
& =\left[f,\left[f_{\lambda \mid \mu},\left[f_{\mu}, g_{\nu}\right]\right]\right] \\
& =\left[f,\left[f_{\lambda \mid \mu} \cdot f_{\mu}, g_{\nu}\right]\right] \\
& =\left[f,\left[f_{\lambda}, g_{\nu}\right]\right] .
\end{aligned}
$$

This derivation yields the desired identity, since Proposition 4.7 yields

$$
\left[f_{\lambda}, g_{\nu}\right]= \begin{cases}g_{\nu \mid \lambda} & \text { if } \lambda \preceq \nu, \\ 0 & \text { if } \lambda \npreceq \nu .\end{cases}
$$

The preceding result is used to obtain the following down-recurrence, defining a basis in terms of basis functions of lower degree.

Corollary 4.10 (Down recurrence for (relative) dual basis functions). Under the assumptions stated at the beginning of this section, let $0 \leq m \leq n$, and consider multi-indices $\mu \in \mathcal{I}_{s, m}$ and $\nu \in \mathcal{I}_{s, n}$, with $\mu \preceq \nu$.

1. For $m \leq k \leq n$,

$$
g_{\nu \mid \mu}=\sum_{\lambda} g_{\nu \mid \lambda} g_{\lambda \mid \mu}
$$

where the summation ranges over all indices $\lambda \in \mathcal{I}_{s, k}$ with $\mu \preceq \lambda \preceq \nu$.

2. For $\xi^{1}, \ldots, \xi^{k} \in \mathbb{R}^{s}$, with $m+k \leq n$,

$$
D_{\xi^{1}} \cdots D_{\xi^{k}} g_{\nu \mid \mu}(x)=\frac{(n-m) !}{(n-m-k) !} \sum_{\lambda} G_{\nu \mid \lambda}\left(\xi^{1}, \ldots, \xi^{k}\right) g_{\lambda \mid \mu}(x),
$$

where $G_{\nu \mid \lambda}$ is the polar form of $g_{\nu \mid \lambda} \in \mathcal{H}_{k}\left(\mathbb{R}^{s}\right)$, and the summation ranges over all indices $\lambda \in \mathcal{I}_{s, n-k}$ with $\mu \preceq \lambda \preceq \nu$. In particular, the $k$-th derivative in direction $z \in \mathbb{R}^{s}$ is

$$
D_{z}^{k} g_{\nu \mid \mu}(x)=\frac{(n-m) !}{(n-m-k) !} \sum_{\lambda} g_{\nu \mid \lambda}(z) g_{\lambda \mid \mu}(x) .
$$

Proof. The first claim follows by taking $f=1 \in \mathcal{H}_{0}\left(\mathbb{R}^{s}\right)$ in Proposition 4.9 To prove the second part, take $f=\left(\xi^{1}, \cdot\right) \cdots\left(\xi^{k}, \cdot\right)$ in Proposition 4.9 Applying Corollary 2.3 part 1 , to $g_{\nu \mid \mu} \in \mathcal{H}_{n-m}\left(\mathbb{R}^{s}\right)$, we see that

$$
\left[f, g_{\nu \mid \mu}\right]=\frac{(n-m-k) !}{(n-m) !} D_{\xi^{1}} \cdots D_{\xi^{k}} g_{\nu \mid \mu}
$$

Similarly, for $\lambda \in \mathcal{I}_{s, n-k}$ we have $g_{\nu \mid \lambda} \in \mathcal{H}_{k}\left(\mathbb{R}^{s}\right)$, so according to Proposition 2.8 we have $\left[f, g_{\nu \mid \lambda}\right]=G_{\nu \mid \lambda}$, which proves the first identity of the second part. Diagonalizing, i.e., taking $\xi^{1}=\ldots=\xi^{k}=z$, completes the proof. 
Down recurrence for $B$-patches. Let $\mathcal{X}$ be an $s \times n$ array of points satisfying the transversal basis property. Taking $m=0$ and $k=n-1$ in Corollary 4.10 part 1 , yields $b_{\alpha}(x)=\sum_{i=1}^{s} g_{\alpha \mid \alpha-e_{i}}(x) b_{\alpha-e_{i}}(x)$, for $\alpha \in \Gamma_{s, n}$ and $x \in \mathbb{R}^{s}$, where we adopt the convention that $g_{\alpha \mid \alpha-e_{i}}=0$ and $b_{\alpha-e_{i}}=0$ if $\alpha_{i}=0$. It follows from Definition 4.1 that $g_{\alpha \mid \alpha-e_{i}}=u_{i, \alpha-e_{i}}$, so we get the following recurrence for B-patches:

$$
b_{\alpha}(x)=\sum_{i=1}^{s} u_{i, \alpha-e_{i}}(x) b_{\alpha-e_{i}}(x) .
$$

Similarly, taking $k=1, m=0$, and $\xi^{1}=z \in \mathbb{R}^{s}$, the identity in part 2 of Corollary 4.10 translates into

$$
D_{z} b_{\alpha}(x)=n \sum_{i=1}^{s} u_{i, \alpha-e_{i}}(z) b_{\alpha-e_{i}}(x)
$$

For a different derivation of these recurrences we again refer to [6] and [17].

Down recurrence for knot-patches. To obtain a similar recurrence for knot-patches, consider a set $X$ consisting of $n+s-1$ points of $\mathbb{R}^{s}$ in general position. Taking again $m=0$ and $k=n-1$ in Corollary 4.10, we get the following recurrence for the knot-patch $N_{I}$, for $I \in \Lambda_{s, n}$ :

$$
N_{I}(x)=\sum_{\substack{J \in \Lambda_{s, n-1} \\ J \preceq I}} N_{I \mid J}(x) N_{J}(x) .
$$

Observe that the summation index $J$ is a subset of $\{1, \ldots,(n-1)+(s-1)\} \cap I$ of cardinality $n-1$, so $J$ is of the form $I-\{i\}$, where $i \in I$ and $i \neq n+s-1$.

For $I \in \Lambda_{s, n}$, let $\left\{u_{i, I} \mid i \in\{1, \ldots, n+s-1\}-I\right\}$ be the basis of $\mathcal{H}_{1}\left(\mathbb{R}^{s}\right)$ consisting of the linear functions dual to $\left\{x_{i} \mid i \in\{1, \ldots, n+s-1\}-I\right\}$, i.e., $u_{i, I}\left(x_{j}\right)=\delta_{i j}$ for $i, j \in\{1, \ldots, n+s-1\}-I$. Then the recurrence for $N_{I}$ boils down to

$$
N_{I}(x)=\sum_{\substack{i \in I \\ i \neq n+s-1}} u_{i, I-\{i\}}(x) N_{I-\{i\}}(x) .
$$

In a completely similar way we obtain the following recurrence for the derivatives of knot-patches in the direction $z \in \mathbb{R}^{s}$ :

$$
D_{z} N_{I}(x)=n \sum_{\substack{i \in I \\ i \neq n+s-1}} u_{i, I-\{i\}}(z) N_{I-\{i\}}(x) .
$$

4.4. Upward recurrence: de Boor/de Casteljau's algorithm. de Casteljau's algorithm for the evaluation of a point on a polynomial curve in Bézier form is one of the basic algorithms in computer aided geometric design; see, e.g., [10]. Using the machinery developed in this paper we derive, more or less routinely, a generalized version of this algorithm in the context of order-compatible bases.

Again we adopt the notation and assumptions stated at the beginning of Section 4.3. In this setting, the generalized de Casteljau's algorithm takes as input a 
collection of scalars $c_{\nu} \in \mathbb{R}$, for $\nu \in \mathcal{I}_{s, n}$, and $n$ points $\xi^{1}, \ldots, \xi^{n} \in \mathbb{R}^{s}$. It evaluates the polar form of the homogeneous polynomial

$$
h=\sum_{\nu \in \mathcal{I}_{s, n}} c_{\nu} g_{\nu}
$$

at $\left(\xi^{1}, \ldots, \xi^{n}\right)$. The classical de Casteljau algorithm solves this problem in case $\mathcal{G}_{n}$ is the homogeneous Bernstein-Bézier basis of $\mathcal{H}_{n}\left(\mathbb{R}^{s}\right)$, and $\xi^{1}=\ldots=\xi^{n}=x \in \mathbb{R}^{s}$.

The generalized algorithm computes, for $m$ ranging over an increasing sequence

$$
0=m_{0}<m_{1}<\ldots<m_{t}=n,
$$

the polar forms $\mathcal{P}^{m}\left[f_{\mu}, h\right]$, for all $\mu \in \mathcal{I}_{s, n-m}$. For $m=0$ these polar forms correspond to the input values $c_{\mu}, \mu \in \mathcal{I}_{s, n}$, since in this case

$$
\mathcal{P}^{0}\left[f_{\mu}, h\right]=\left[f_{\mu}, h\right]=c_{\mu} .
$$

For $m=n$, the single basis function in $\mathcal{F}_{0}$ is $1 \in \mathcal{H}_{0}\left(\mathbb{R}^{s}\right)$, according to the assumptions stated at the beginning of Section 4.3 Therefore the corresponding polar form is $\mathcal{P}^{n}[1, h]=\mathcal{P}^{n} h$, i.e., the desired output.

It remains to describe the basic step of the algorithm, where $m$ is increased from $m=m_{j-1}$ to $m=m_{j}$, for $0<j \leq n$. For convenience we denote $\mathcal{P}^{m}\left[f_{\mu}, h\right]$ by $C_{\mu}^{m}$, for $\mu \in \mathcal{I}_{s, n-m}$. In particular, $C_{\mu}^{m}$ is a symmetric $m$-linear form on $\mathbb{R}^{s}$. Assume we have determined $C_{\mu}^{m}\left(\xi^{1}, \ldots, \xi^{m}\right)$, for all $\mu \in \mathcal{I}_{s, n-m}$. Our goal is to determine $C_{\lambda}^{m+k}\left(\xi^{1}, \ldots, \xi^{m+k}\right)$, for all $\lambda \in \mathcal{I}_{s, n-m-k}$. Since

$$
C_{\lambda}^{m+k}\left(\xi^{1}, \ldots, \xi^{m+k}\right)=\left[\left(\xi^{1}, \cdot\right) \cdots\left(\xi^{m+k}, \cdot\right),\left[f_{\lambda}, h\right]\right]
$$

transposition of a factor $\left(\xi^{m+1}, \cdot\right) \cdots\left(\xi^{m+k}, \cdot\right)$, cf. Theorem 2.2 part 3 , yields

$$
C_{\lambda}^{m+k}\left(\xi^{1}, \ldots, \xi^{m+k}\right)=\left[\left(\xi^{1}, \cdot\right) \cdots\left(\xi^{m}, \cdot\right),\left[\left(\xi^{m+1}, \cdot\right) \cdots\left(\xi^{m+k}, \cdot\right) f_{\lambda}, h\right]\right]
$$

Suppose we have expressed the polynomial $\left(\xi^{m+1}, \cdot\right) \cdots\left(\xi^{m+k}, \cdot\right) f_{\lambda} \in \mathcal{H}_{n-m}\left(\mathbb{R}^{s}\right)$ with respect to the basis $\left\{f_{\mu} \mid \mu \in \mathcal{I}_{s, n-m}\right\}$ as

$$
\left(\xi^{m+1}, \cdot\right) \cdots\left(\xi^{m+k}, \cdot\right) f_{\lambda}=\sum_{\mu} a_{\mu} f_{\mu},
$$

where $\mu$ ranges over $\mathcal{I}_{s, n-m}$. Then the right hand side of (4.8) reduces to

$$
\begin{aligned}
\sum_{\mu}\left[\left(\xi^{1}, \cdot\right) \cdots\left(\xi^{m}, \cdot\right),\left[a_{\mu} f_{\mu}, h\right]\right] & =\sum_{\mu} a_{\mu}\left[\left(\xi^{1}, \cdot\right) \cdots\left(\xi^{m}, \cdot\right),\left[f_{\mu}, h\right]\right] \\
& =\sum_{\mu} a_{\mu} C_{\mu}^{m}\left(\xi^{1}, \ldots, \xi^{m}\right) .
\end{aligned}
$$

In view of Proposition 3.4 part 1 , the scalar $a_{\mu}$ in (4.9) satisfies

$$
\begin{aligned}
a_{\mu} & =\left[\left(\xi^{m+1}, \cdot\right) \cdots\left(\xi^{m+k}, \cdot\right) f_{\lambda}, g_{\mu}\right] \\
& =\left[\left(\xi^{m+1}, \cdot\right) \cdots\left(\xi^{m+k}, \cdot\right),\left[f_{\lambda}, g_{\mu}\right]\right] \\
& = \begin{cases}G_{\mu \mid \lambda}\left(\xi^{m+1}, \ldots, \xi^{m+k}\right), & \text { if } \lambda \preceq \mu, \\
0, & \text { if } \lambda \npreceq \mu,\end{cases}
\end{aligned}
$$

where $G_{\mu \mid \lambda}\left(\xi^{m+1}, \ldots, \xi^{m+k}\right)$ is the polar form of $\left[f_{\lambda}, g_{\mu}\right]=g_{\mu \mid \lambda}$, evaluated at $\left(\xi^{m+1}, \ldots, \xi^{m+k}\right)$ in case $\lambda \preceq \mu$ (cf. Proposition 4.7). Therefore the summation in (4.10) ranges over the subset $\mathcal{I}_{s, k}(\lambda)$ of $\mathcal{I}_{s, n-m}$. Summarizing, we get 
Lemma 4.11 (Upward recurrence). Let $n \geq 0$. Under the assumptions stated at the beginning of Section 4.3, let $c_{\nu} \in \mathbb{R}, \nu \in \mathcal{I}_{s, n}$, be the coefficients of the polynomial $h=\sum_{\nu \in \mathcal{I}_{s, n}} c_{\nu} g_{\nu}$ with respect to $\mathcal{G}_{n}$. Let $\xi^{1}, \ldots, \xi^{n} \in \mathbb{R}^{s}$, and let $C_{\mu}^{m}$ be the polar form of $\left[f_{\mu}, h\right]$.

Furthermore, let $0 \leq m<m+k \leq n$ and, for all $\lambda \in \mathcal{I}_{s, n-m-k}$ and $\mu \in \mathcal{I}_{s, k}(\lambda)$, let $G_{\mu \mid \lambda}$ be the polar form of the basis function $g_{\mu \mid \lambda} \in \mathcal{H}_{k}\left(\mathbb{R}^{s}\right)$. Then

$$
C_{\lambda}^{m+k}\left(\xi^{1}, \ldots, \xi^{m+k}\right)=\sum_{\mu} C_{\mu}^{m}\left(\xi^{1}, \ldots, \xi^{m}\right) G_{\mu \mid \lambda}\left(\xi^{m+1}, \ldots, \xi^{m+k}\right),
$$

where the summation ranges over all indices $\mu \in \mathcal{I}_{s, k}(\lambda)$.

This upward recurrence relation yields a class of de Casteljau-like algorithms for computing the polar form of $h$, evaluated at $\left(\xi^{1}, \ldots, \xi^{m}\right)$, by computing the families $\left\{C_{\mu}^{m}\left(\xi^{1}, \ldots, \xi^{m}\right) \mid \mu \in \mathcal{I}_{s, n-m}\right\}$, where $m$ runs from $m=0$ to $m=n$ in arbitrary steps. Taking the step size e.g. equal to 1 , we get the following result.

Corollary 4.12 (Generalized de Casteljau/de Boor Algorithm). Let $n \geq 0$. Under the assumptions stated at the beginning of Section 4.3, let $c_{\nu} \in \mathbb{R}, \nu \in \mathcal{I}_{s, n}$, be the coefficients of the polynomial $h=\sum_{\nu \in \mathcal{I}_{s, n}} c_{\nu} g_{\nu}$ with respect to $\mathcal{G}_{n}$, and let $\xi^{1}, \ldots, \xi^{n} \in \mathbb{R}^{s}$. Let $C_{\mu}^{m}\left(\xi^{1}, \ldots, \xi^{m}\right)$, with $\mu \in \mathcal{I}_{s, n-m}$, be defined as follows:

1. For all $\nu \in \mathcal{I}_{s, n}$,

$$
C_{\nu}^{0}()=c_{\nu}
$$

2. For $0 \leq m<n$, and $\lambda \in \mathcal{I}_{s, n-m-1}$,

$$
C_{\lambda}^{m+1}\left(\xi^{1}, \ldots, \xi^{m+1}\right)=\sum_{\mu} C_{\mu}^{m}\left(\xi^{1}, \ldots, \xi^{m}\right) g_{\mu \mid \lambda}\left(\xi^{m+1}\right),
$$

where the summation is over all indices $\mu \in \mathcal{I}_{s, n-m}$ with $\lambda \preceq \mu$.

Then $C_{\mu}^{m}$ is a symmetric m-linear form on $\mathbb{R}^{s}$, equal to the polar form of $\left[f_{\mu}, h\right]$, for $\mu \in \mathcal{I}_{s, n-m}$. In particular, $C_{0}^{n}\left(\xi^{1}, \ldots, \xi^{n}\right)$ is the polar form of $h$, evaluated at $\left(\xi^{1}, \ldots, \xi^{n}\right)$.

Note that there are exactly $s$ indices in the summation range of the recurrence (4.11); see Section 4.2.

The up-recurrence in Corollary 4.12 is also derived in Micchelli [17, Section 5.6] and Cavaretta and Micchelli [4] for the special case of a polynomial in B-form. This case is elaborated in one of the examples later in this section. In the latter papers the fact that $C_{\mu}^{m}$ is symmetric requires a much longer proof. In our approach this symmetry comes for free with our characterization of $C_{\mu}^{m}$ as the polar form of $\left[f_{\mu}, h\right]$.

Application of Corollary 3.5 yields the following decomposition of the $m$-th order derivative of $h$ with respect to the basis $\mathcal{G}_{n-m}$ of $\mathcal{H}_{n-m}\left(\mathbb{R}^{s}\right)$ :

$$
D_{\xi^{1}} \cdots D_{\xi^{m}} h=\frac{n !}{(n-m) !} \sum_{\mu \in \mathcal{I}_{s, n-m}} C_{\mu}^{m}\left(\xi^{1}, \ldots, \xi^{m}\right) g_{\mu} .
$$

See also (4.6) in the proof of Proposition 4.8. Therefore $m$ steps of the generalized de Casteljau/de Boor algorithm are sufficient to compute the $m$-th order derivative of $h$ (in the mixed directions $\xi^{1}, \ldots, \xi^{m}$ ). 
The classical de Casteljau algorithm for evaluation of a polynomial in BernsteinBézier form at a point $x \in \mathbb{R}^{s}$ is obtained by taking for $\mathcal{G}_{n}$ in Corollary 4.12 the Bernstein-Bézier basis of $\mathcal{H}_{n}\left(\mathbb{R}^{s}\right)$, and diagonalizing, i.e., taking $\xi^{1}=\ldots=\xi^{n}=x$. The derivation of de Boor's algorithm for the evaluation of a polynomial in B-form is illustrated in the following example.

Application: Up-recurrence for B-patches. We describe the basic step in the generalized de Casteljau/de Boor algorithm, applied to a polynomial in B-form. So let $\mathcal{X}$ be an $s \times n$ array of points in $\mathbb{R}^{s}$, satisfying the transversal basis property. In particular, consider $h \in \mathcal{H}_{n}\left(\mathbb{R}^{s}\right)$ with decomposition $h=\sum_{\nu \in \Gamma_{s, n}} c_{\nu} b_{\nu} \in \mathcal{H}_{n}\left(\mathbb{R}^{s}\right)$ with respect to the B-patch basis $\mathcal{B}_{n}(\mathcal{X})$ of $\mathcal{H}_{n}\left(\mathbb{R}^{s}\right)$, corresponding to $\mathcal{X}$. Then the polar form of $h$ can be evaluated at $\left(\xi^{1}, \ldots, \xi^{m+1}\right)$ by the following recurrence:

$$
C_{\lambda}^{m+1}\left(\xi^{1}, \ldots, \xi^{m+1}\right)=\sum_{i=1}^{s} C_{\lambda+e_{i}}^{m}\left(\xi^{1}, \ldots, \xi^{m}\right) u_{i, \lambda}\left(\xi^{m+1}\right)
$$

starting from $C_{\nu}^{0}()=c_{\nu}$, for $\nu \in \Gamma_{s, n}$. In particular, $h(x)=C_{0}^{n}(x, \ldots, x)$. As indicated above, the linear form $C_{\lambda}^{m}$ is $m$-symmetric, since it is the polar form of $\left[l_{\lambda}, h\right]$.

Application: Conversion between B-patch bases. An interesting application of the generalized de Casteljau/de Boor algorithm is the conversion between representations of a polynomial with respect to two different B-patch bases, as described, e.g., in Lodha and Goldman [15].

Consider the setting of Section 4.1.2 in which two $s \times n$ arrays $\mathcal{X}$ and $\overline{\mathcal{X}}$ only differ in their last rows, i.e., $x^{i, j}=\bar{x}^{i, j}$, for $1 \leq i<s$. Both arrays are assumed to satisfy the transversal basis property. Let $h=\sum_{\nu \in \Gamma_{s, n}} c_{\nu} b_{\nu}$ be the representation of a polynomial $h \in \mathcal{H}_{n}\left(\mathbb{R}^{s}\right)$ with respect to the B-patch basis $\mathcal{B}_{n}(\mathcal{X})$. Then our goal is to compute the representation $h=\sum_{\nu \in \Gamma_{s, n}} \bar{c}_{\nu} \bar{b}_{\nu}$ with respect to the lineal basis $\mathcal{B}_{n}(\overline{\mathcal{X}})$.

Since $\bar{c}_{\nu}=\left[\bar{l}_{\nu}, h\right]$, we obtain, by isolating linear factors corresponding to the last row of $\overline{\mathcal{X}}$

$$
\begin{aligned}
\bar{c}_{\nu}=\left[\bar{l}_{\nu}, h\right] & =\left[l_{\nu-\nu_{s} e_{s}} \bar{l}_{\nu_{s} e_{s}}, h\right] \\
& =\left[\bar{l}_{\nu_{s} e_{s}},\left[l_{\nu-\nu_{s} e_{s}}, h\right]\right] \\
& =C_{\nu-\nu_{s} e_{s}}^{\nu_{s}}\left(\bar{x}^{s, 1}, \ldots, \bar{x}^{s, \nu_{s}}\right) .
\end{aligned}
$$

In other words, the coefficient $\bar{c}_{\nu}$ of $h$ with respect to the basis $\mathcal{B}_{n}(\overline{\mathcal{X}})$ is obtained by running the generalized de Casteljau/de Boor algorithm to evaluate the polar form of $h$ at the points in the last row of $\overline{\mathcal{X}}$. The coefficient $\bar{c}_{\nu}$ is the entry in the "de Casteljau simplex', at the position corresponding to the multi-index $\nu-\nu_{s} e_{s}$. Note that the latter multi-index lies in the face of the de Casteljau-simplex consisting of all multi-indices $\mu$ with last entry equal to zero, i.e., with $\mu_{s}=0$.

The general change of basis algorithm is obtained by replacing the rows of $\mathcal{X}$ by those of $\overline{\mathcal{X}}$ one by one; see also Section 4.1.2. It should be noted that the lineal change of basis algorithm, presented in Section 4.1.2 is derived in Lodha and Goldman [15] from the above algorithm for conversion between B-patch bases, using duality. 
Application: Up-recurrence for knot patches. Finally, consider a set $X$, consisting of $n+s-1$ points in $\mathbb{R}^{s}$ in general position. Let $h \in \mathcal{H}_{n}\left(\mathbb{R}^{s}\right)$ have decomposition $h=\sum_{I \in \Lambda_{s, n}} c_{I} N_{I} \in \mathcal{H}_{n}\left(\mathbb{R}^{s}\right)$ with respect to the knot-patch basis of $\mathcal{H}_{n}\left(\mathbb{R}^{s}\right)$, corresponding to $X$ (cf. Section 4.1.3). Then the polar form of $h$ can be evaluated at $\left(\xi^{1}, \ldots, \xi^{m+1}\right)$ by the following recurrence:

$$
C_{I}^{m+1}\left(\xi^{1}, \ldots, \xi^{m+1}\right)=\sum_{i \in\{1, \ldots, n-m+s-1\}-I} C_{I+\{i\}}^{m}\left(\xi^{1}, \ldots, \xi^{m}\right) u_{i, I}\left(\xi^{m+1}\right),
$$

starting from $C_{I}^{0}()=c_{I}$, for $I \in \Lambda_{s, n}$. Also in this case, $h(x)=C_{0}^{n}(x, \ldots, x)$.

\section{Future RESEARCH}

In a forthcoming paper the theory of order compatible bases, developed in Section 4, will be applied to compute a basis for the solution space of constant coefficient PDE's. The computations to be presented will be based on the following observations. Consider the partial differential equation $p(\partial) f=0$, where $p \in \mathcal{H}_{m}\left(\mathbb{R}^{s}\right)$ is a fixed polynomial (e.g. $p=x_{1}^{2}+\cdots+x_{s}^{2}$, in which case the PDE is the Laplace equation). Solving this PDE for $f \in \mathcal{H}_{n}\left(\mathbb{R}^{s}\right), n \geq m$, amounts to finding the kernel of the linear operator $D_{p}: \mathcal{H}_{n}\left(\mathbb{R}^{s}\right) \rightarrow \mathcal{H}_{n-m}\left(\mathbb{R}^{s}\right)$, defined by $D_{p}(f)=[p, f]$, cf. Definition 2.1 It is not hard to show that this operator is the adjoint - with respect to apolar pairing — of the multiplication operator $T_{p}: \mathcal{H}_{n-m}\left(\mathbb{R}^{s}\right) \rightarrow \mathcal{H}_{n}\left(\mathbb{R}^{s}\right)$, defined by $T_{p}(f)=p f$. Therefore, we have the decomposition $\mathcal{H}_{n}\left(\mathbb{R}^{s}\right)=\operatorname{Ker} D_{p} \oplus \operatorname{Im} T_{p}$, which is an orthogonal sum decomposition with respect to the apolar inner product on $\mathcal{H}_{n}\left(\mathbb{R}^{s}\right)$. This property was also observed by Reznick [22] and 23], and by Beauzamy et al. 11. In 24 these techniques are applied to obtain normal forms for the polynomial solutions of constant (complex) coefficient partial differential equations. Pedersen [19] applies similar techniques in the case of a PDE with real coefficients, and to a system of PDE's in [20. Our forthcoming paper will exploit properties of order compatible and dual bases to construct the solution space efficiently.

We further intend to extend our work to obtain simple algorithms for, e.g., degree reduction and degree elevation of multivariate Bernstein-Bézier patches and B-patches, using the apolar inner product to define projections onto subspaces corresponding to polynomials of lower degree.

\section{ACKNOWLEDGMENTS}

I wish to thank Lyle Ramshaw for his interest in this paper, and the anonymous referee for suggesting a useful addition to the introductory section.

\section{REFERENCES}

1. B. Beauzamy and J. Dégot, Differential identities, Transactions Amer. Math. Society 347 (1995), 2607-2619. MR 96c:05009

2. P. de Casteljau, Formes à pôles, Hermes, Paris, 1985.

3.

4. A.S. Cavaretta and C.A. Micchelli, Pyramid patches provide potential polynomial paradigms, Mathematical methods in CAGD and image processing (New York), Academic Press, 1992, pp. 69-100. MR 93h:65023

5. H.B. Curry and I.J. Schoenberg, On Pólya frequency functions IV: The fundamental spline functions and their limits, Journal d'Analyse Math. 17 (1966), 71-107. [MR 36:1884]

6. W. Dahmen, C.A. Micchelli, and H.-P. Seidel, Blossoming begets B-spline bases built better by B-patches, Mathematics of Computation 59 (1992), 97-115. MR 93b:41014 
7. C. de Boor, A practical guide to splines, Appl. Math. Sciences, vol. 27, Springer Verlag, New York, 1978. MR 80a:65027

8. R.A. DeVore and G.G. Lorentz, Constructive approximation, Grundlehren der mathematischen Wissenschaften, vol. 303, Springer-Verlag, Berlin, 1993. MR 95f:41001

9. R. Ehrenborg and G.-C. Rota, Apolarity and canonical forms for homogeneous polynomials, European J. Combinatorics 14 (1993), 157-191. MR 94e:15062

10. G. Farin, Curves and surfaces for CAGD. a practical guide, Computer science and scientific computing, Academic Press, Inc., Boston, 1993. MR 97e:65022 (later ed.)

11. Ph. Fong and H.-P. Seidel, An implementation of B-spline surfaces over arbitrary triangulations, Computer Aided Geometric Design 10 (1993), 267-275. MR 94k:65017

12. R.N. Goldman, Dual polynomial bases, J. Approx. Theory 79 (1994), 311-346. MR 95i:41013

13. J.P.S. Kung and G.-C. Rota, The invariant theory of binary forms, Bull. Amer. Math. Soc. 10 (1984), 27-85. MR 85g:05002

14. S. Lodha and R. Goldman, A multivariate generalization of the de Boor-Fix formula, Curves and Surfaces in Geometric Design (G. Farin, ed.), A.K. Peters, Wellesley, MA, 1994, pp. 301310. MR 95g:65026

15. Change of basis algorithms for surfaces in CAGD, Computer Aided Geometric Design 12 (1995), 801-824. MR 97b:65025

16. M.J. Marsden, An identity for spline functions with applications to variation-diminishing spline approximations, J. Approx. Theory 3 (1970), 7-49. MR 40:7682

17. C.A. Micchelli, Mathematical aspects of geometric modeling, Regional conference series in applied mathematics, vol. 65, Siam, Philadelphia, 1995. MR 95i:65036

18. M. Neamtu, Homogeneous simplex splines, J. Comput. Appl. Math. 73 (1996), 173-189. MR 97h:41025

19. P.S. Pedersen, A function theory for finding a basis for all polynomial solutions to linear constant coefficient PDE's of homogeneous order, Complex Variables Theory Applications 24 (1993), 79-87. MR 95b:30081

20. _ A basis for polynomial solutions to systems of linear constant coefficient PDE's, Advances in Mathematics 117 (1996), 157-163. MR 96k:35018

21. L. Ramshaw, Blossoms are polar forms, Computer Aided Geometric Design 6 (1989), 323-358. MR 91d:65026

22. B. Reznick, Sums of even powers of real linear forms, Memoirs Amer. Math. Soc., vol. 96, no. 463, AMS, Providence, RI, 1992. MR 93h:11043

23. $ـ$ An inequality for products of polynomials, Proc. Amer. Math. Society 117 (1993), 1063-1073. MR 93e:11058

24. Homogeneous polynomial solutions to constant coefficient PDE's, Advances in Mathematics 117 (1996), 179-192. MR 97a:12006

25. L.L. Schumaker, Spline functions: Basic theory, John Wiley \& Sons, New York, 1981. MR 82j:41001

Department of Mathematics and Computing Science, University of Groningen, P.O. Box 800, 9700 AV Groningen, The Netherlands

E-mail address: gert@cs.rug.nl

$U R L:$ http://www.cs.rug.nl/ ${ }^{\sim}$ gert 